\title{
Constraining the parameter space of the one-zone synchrotron-self-Compton model for GeV-TeV detected BL Lacertae objects
}

\author{
M. Cerruti ${ }^{1,2}$, C. Boisson ${ }^{1}$, and A. Zech ${ }^{1}$ \\ ${ }^{1}$ LUTH, Observatoire de Paris, CNRS, Université Paris Diderot, 5 place Jules Janssen, 92190 Meudon, France \\ ${ }^{2}$ Harvard-Smithsonian Center for Astrophysics, 60 Garden Street, Cambridge MA, 02138, USA \\ e-mail: matteo.cerruti@cfa.harvard.edu
}

Received 20 December 2012 / Accepted 19 May 2013

\section{ABSTRACT}

\begin{abstract}
Context. The one-zone synchrotron-self-Compton (SSC) model aims to describe the spectral energy distribution (SED) of BL Lac objects via synchrotron emission by a non-thermal population of electrons and positrons in a single homogeneous emission region, partially upscattered to $\gamma$-rays by the particles themselves.

Aims. The model is usually considered as degenerate, given that the number of free parameters is higher than the number of observables. It is thus common to model the SED by choosing a single set of values for the SSC model parameters that provide a good description of the data, without studying the entire parameter space. We present here a new numerical algorithm that permits us to find the complete set of solutions, using the information coming from the detection in the $\mathrm{GeV}$ and $\mathrm{TeV}$ energy bands.

Methods. The algorithm is composed of three separate steps: we first prepared a grid of simulated SEDs and extracted from each SED the values of the observables; we then parametrized each observable as a function of the SSC parameters; we finally solved the system for a given set of observables. We iteratively solved the system to take into account uncertainties in the values of the observables, producing a family of solutions.

Results. We present a first application of our algorithm to the typical high-frequency-peaked BL Lac object 1RXS J101015.9 - 311909, provide constraints on the SSC parameters, and discuss the result in terms of our understanding of the blazar emitting region.
\end{abstract}

Key words. radiation mechanisms: non-thermal - relativistic processes - methods: numerical - BL Lacertae objects: general galaxies: individual: 1RXS J101015.9-311909

\section{Introduction}

Blazars are a class of active galactic nuclei (AGN) characterised by extreme variability, a high degree of polarization, and a strong non-thermal continuum observed in the optical/UV spectrum (Stein et al. 1976; Moore \& Stockman 1981). According to the unified AGN model, they are considered to be radio-loud AGN with the relativistic jet pointing in the direction of the observer (Urry \& Padovani 1995). Their spectral energy distribution (SED) is thus dominated by the non-thermal emission from the jet, enhanced by relativistic effects. The blazar class is divided into the two subclasses of flat-spectrum radio quasars (FSRQs) and BL Lacertae (BL Lac) objects according to the strength of the non-thermal continuum emission relative to the thermal emission from the accretion disc that is partially reprocessed in the broad-line region (BLR). A blazar is then classified as a BL Lac object if the optical/UV spectrum is dominated by the continuum emission, while it is classified as an FSRQ if emission lines and/or the big blue bump are observed (the standard threshold between the two subclasses being an equivalent width of the emission lines equal to $5 \AA$, Angel \& Stockman 1980).

The blazar SED is composed of two components, the first one peaking between infra-red and X-rays, the second one peaking in $\gamma$-rays (see e.g. Abdo et al. 2010). The position of the peak frequency of the first component is used to differentiate among subclasses of BL Lac objects: we can then identify a
BL Lac object as high-frequency-peaked BL Lac (HBL) if the peak frequency is located in UV/X-rays, or as low-frequencypeaked BL Lac (LBL) if the peak frequency is located in infrared/visible light (see Padovani \& Giommi 1995). The FSRQs always show a low-frequency peak. The position of the first peak frequency seems to be inversely correlated with the luminosity (the so-called blazar sequence, Fossati et al. 1998), even though there is not a general consensus on this point (see e.g. Nieppola et al. 2008; Padovani et al. 2012).

The origin of the first bump is generally ascribed to synchrotron emission from a non-thermal population of electrons and positrons in the emitting region, while the second bump is often ascribed to inverse Compton processes of the same leptons with their own synchrotron emission (synchrotron-self-Compton model, SSC, Konigl 1981) or with an external photon field (external-inverse-Compton, EIC, Dermer et al. 1992; Dermer \& Schlickeiser 1993; Sikora et al. 1994) such as the BLR, the accretion disc or the external torus. While the SSC model successfully describes the SED of HBLs, an external Compton component is required for LBLs and FSRQs (see e.g. Ghisellini et al. 2011).

It is worth recalling that other kinds of models exist, in particular hadronic models in which the emitting region also contains relativistic protons, which are responsible (together with the secondary particles coming from $\mathrm{p}-\gamma$ interactions) for the high energy component of the SED (see e.g. Mannheim 1993; Mücke \& Protheroe 2001). 


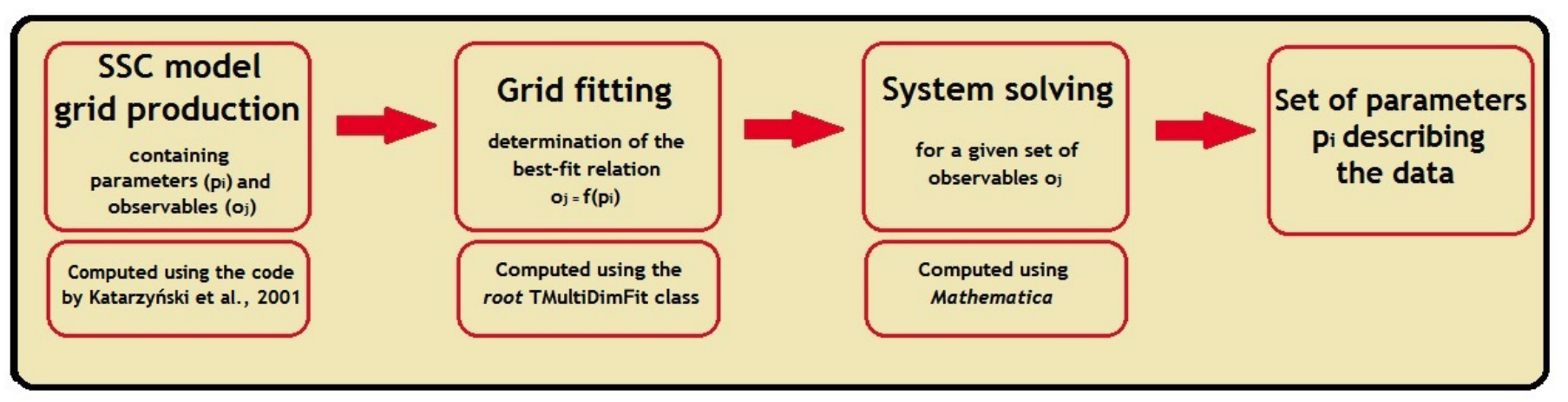

Fig. 1. Flow diagram for the new numerical algorithm for constraining the SSC model parameters.

The one-zone SSC modelling of HBLs is usually considered to be degenerate: the number of free parameters is higher than the number of observational constraints, and a best-fit solution cannot be given. The standard approach is thus to find one set of parameters that successfully describes the SED, without evaluating the uncertainties on these parameters. In this paper we present a new algorithm to constrain the parameter space of the model, using the information from the $\mathrm{GeV}$ and $\mathrm{TeV}$ $\gamma$-ray emission, as observed by Fermi-LAT (Atwood et al. 2009) and ground-based imaging atmospheric Cherenkov telescopes (IACTs), respectively.

Before describing our algorithm, we recall here the basis of the one-zone SSC model by Katarzyński et al. (2001), on which this study is based. The algorithm can easily be adapted to similar models. The emitting region is considered to be a spherical blob of plasma (with radius $R$ ), moving in the relativistic jet with a Doppler factor $\delta$. The source is supposed to be filled with a tangled, homogeneous magnetic field $B$ and a non-thermal population of leptons $\left(\mathrm{e}^{ \pm}\right)$. The particle energy distribution is described as a broken power-law function (a spectral break is expected in the presence of synchrotron radiation, see e.g. Inoue $\&$ Takahara 1996), defined by the two indexes $\alpha_{1}$ and $\alpha_{2}$; the three Lorentz factors $\gamma_{\min }, \gamma_{\text {break }}$, and $\gamma_{\text {Max }}$; and the normalization factor $K$. The free parameters of the model are then nine: three for the emitting region $(R, \delta$, and $B)$ and six for the particle population $\left(\gamma_{\min ; b r e a k ; M a x}, \alpha_{1 ; 2}\right.$, and $\left.K\right)$. In general there are less than nine observables to constrain the parameters of the model, which remains thus degenerate. The basis of the constraints on the SSC model are described in the work by Tavecchio et al. (1998, hereafter T98). The idea is to determine a system of equations linking parameters and observables, and to solve it analytically. In their work, T98 used six observables: the frequency and luminosity of the synchrotron peak, the frequency and luminosity of the Compton peak, and the measured X-ray and $\gamma$-ray spectral slopes $\left(\Gamma_{\mathrm{X}, \gamma}\right.$, linked directly to $\alpha_{1}$ and $\alpha_{2}$ through $\left.\alpha_{1,2}=2 \Gamma_{\gamma, X}-1\right)$. The number of free parameters was reduced from nine to seven by considering a very low value of $\gamma_{\min }$ and a very high value of $\gamma_{\mathrm{Max}}$; additional constraints were added from information about the variability time-scale and from the requirement that the break Lorentz factor be consistent with the one expected from synchrotron cooling.

In this paper, we present a new algorithm to find the complete set of solutions for the interpretation of SEDs of HBLs with a one-zone SSC model. Our method is based on the approach by T98, but is fully numerical. In the next section we provide the details of our algorithm, showing in Sect. 3 a first application to the HBL 1RXS J101015.9 - 311909.

\section{Description of the algorithm}

Our algorithm can be seen as a numerical extension of the work done by T98. The basic idea is to define a set of equations linking SSC parameters and observables that, in our case, are obtained numerically. The algorithm is composed of three separate steps (summarized in Fig. 1):

- we simulate SEDs for a grid of parameter values (spanning the region of interest of the SSC parameters $p_{j}$ ), and for each SED we compute the value of the corresponding observables $\left(O_{i}\right)$

- we then parametrize each observable $O_{i}$ as a function of the SSC parameters

$\log O_{i}=\sum_{j} \alpha_{j} \log p_{j}$,

where $\alpha_{j}$ is the result of a fit;

- we finally solve the set of equations for a given set of observables $O_{i}$, to obtain the solution of our model $p_{j}$. If the observables $O_{i}$ include an intrinsic uncertainty $\left(\sigma_{i}\right)$, we iteratively solve the system for $O_{i} \in\left[O_{i}-\sigma_{i}, O_{i}+\sigma_{i}\right]$, thus producing a set of possible solutions.

We reduce the number of free parameters from nine to seven by fixing a reasonably low and high value for $\gamma_{\min }$ (fixed to 100) and $\gamma_{\text {Max }}$ (fixed to $5 \times 10^{6}$ ), respectively. ${ }^{1}$ The main difference with respect to the work by T98 lies in the choice of the observables. The synchrotron peak is relatively well constrained. Even when it is located in the observational gap between the ultra-violet and $\mathrm{X}$-ray bands, its position and luminosity can be reasonably well estimated by extrapolating the low and high energy data. The position of the inverse Compton peak is, however, much more uncertain, and so we decided to use the actually observed $\mathrm{GeV}$ and $\mathrm{TeV}$ spectral slopes, and their flux normalizations, defined in the following as $\Gamma_{\mathrm{GeV}}, \Gamma_{\mathrm{TeV}}, v F_{v ; \mathrm{GeV}}$, and $v F_{v ; \mathrm{TeV}}$. The number of observables we use is thus seven: together with the four $\gamma$-ray observables, we consider the frequency and intensity of the synchrotron peak $\left(v_{\mathrm{s}}\right.$ and $\left.v F_{v ; \mathrm{s}}\right)$, and the measured X-ray spectral slope $\left(\Gamma_{\mathrm{X}}\right)$. We thus have seven free parameters and seven independent observables, and the problem can be solved.

It is important to underline that the four $\gamma$-ray observables are not degenerate: the shape of the inverse Compton component is not symmetrical with respect to its peak, given the fact that

\footnotetext{
These values are consistent with the ones adopted by T98. For the particular case of 1RXS J101015.9 - 311909 presented in the next section, values of $\gamma_{\min }$ lower than 100 would overestimate the radio measurements.
} 
the $\mathrm{TeV}$ part is affected by the transition to the Klein-Nishina regime, the internal absorption by $\gamma-\gamma$ pair production (which depends on the synchrotron emission, see e.g. Aharonian et al. 2008) and the absorption on the extra-galactic background light (EBL, Salamon \& Stecker 1998). It is clear that a study of this kind is carried out more easily with a numerical approach (a purely theoretical derivation of the expression of the TeV spectral slope assuming all the effects described above would be nontrivial). Another improvement with respect to the work by T98 is that we do not use the simple approximation that the $\mathrm{GeV}$ slope is uniquely related to the electron spectral index below the break (when $\gamma_{\min }$ is reasonably low): this approximation is true only if the inverse Compton component accounts for the $\mathrm{GeV}$ spectrum well before the peak; when the Compton peak is located at lower energies, the spectrum measured with Fermi-LAT is significantly softer.

In the SSC code used for this study (Katarzyński et al. 2001), a modification has been introduced concerning the definition of the normalization factor $K$ of the electron distribution, which is originally defined as the number density at $\gamma=1$. With this definition, the value of $K$ depends strongly on the value of $\alpha_{1}$ : in particular, assuming that the model correctly fits the synchrotron peak, but we want to modify $\alpha_{1}$, this imposes a correction of the value of $K$ as well. For a simulation of a multitude of SEDs, this definition is not appropriate, imposing a huge range of values for $K$. We thus redefine $K$ as the number density at $\gamma=\gamma_{\text {break, }}$,

$K^{\prime}=K \gamma_{\text {break }}^{-\alpha_{1}}$.

In this way, if the model correctly describes the synchrotron peak, one can modify the value of $\alpha_{1}$ without affecting the other parameters and, more importantly, the range of values of $K^{\prime}$ to study becomes narrower. As another small modification, the EBL absorption is computed using the model by Franceschini et al. (2008).

In order to minimize the computing time, the code has been parallelized using OpenMP ${ }^{2}$. The parallelization is not at the level of the computation of the synchrotron and inverse Compton components, but at the higher level of the sampling of the parameter space. The distribution of the input parameters per thread is done dynamically, and the communication between threads takes place only at the end of each computation when the results are merged.

Iterating on the values of $\delta, B, K^{\prime}, R, \gamma_{\text {break }}$, and $\alpha_{1}$, SEDs have been simulated for a grid of parameter values (grid production in Fig. 1). The value of $\alpha_{2}$ is fixed, constrained by the measured $X$-ray spectral slope $\Gamma_{X}$, and equal to $2 \Gamma_{X}-1$. For each modelled SED, we first identify the position of the synchrotron peak and its intensity. To compute the expected fluxes and spectral slopes detected in the $\mathrm{GeV}$ and $\mathrm{TeV}$ ranges, we simply fit the modelled SED in the range of the GeV and TeV detection with a power-law function, using as minimum and maximum energies the values adopted in the spectral fitting of the FermiLAT and IACT detection. This defines the two indexes $\Gamma_{\mathrm{GeV} ; \mathrm{TeV}}$, and the two fluxes (of the fitted power-law, not of the model) $v F_{v ; \mathrm{GeV} ; \mathrm{TeV}}$ at the respective decorrelation energies (which are measured quantities).

For the application that will be presented in the next section, we sampled five different values for each free parameter, resulting in the computation of $5^{6}=15625$ SEDs. As an indication, the computing time on our 16-core machine is roughly one hour. The result of this stage is a grid containing the corresponding set of observables for each set of parameters.

\footnotetext{
2 wWW.openmp.org
}

This grid is then fitted using the six observables as dependent variables, and the six free parameters as independent variables (grid fitting in Fig. 1). While for the synchrotron peak frequency and flux the relation between observables and parameters is simple (and consistent with the analytical expression), for the fluxes and slopes measured in the $\mathrm{GeV}$ and $\mathrm{TeV}$ energy bands, it becomes more complicated. In particular, the simple relation given in Eq. (1) no longer applies, and we need to consider a more complex relation of the form

$\log O_{i}=\sum_{k 1 \ldots k 6} \alpha_{k 1 \ldots k 6} \prod_{j} \log p_{j}{ }^{k 1 \ldots k 6}$.

More explicitly, we have to perform a fit considering all the possible polynomials of our parameters.

The problem is then to find a parametrization for a dependent variable, which is a function of six parameters, without knowing it a priori (nonparametric regression). This problem has been solved using the root software ${ }^{3}$, namely the TMultiDimFit class. This class provides, for each term of the fit function, the fitted coefficient and the weight of the term in the overall fit, providing a listing of the different terms in order of relative importance.

The computing time for this step is a few seconds for the synchrotron observables and for $v F_{v ; \mathrm{GeV} ; \mathrm{TeV}}$, while for the $\mathrm{GeV}$ and $\mathrm{TeV}$ slopes it can be significantly longer, taking up to an hour each if we want to study all the possible polynomial functions (in order to be sure not to miss a high-order polynomial which might play an important role in the fit). The choice of the last polynomial considered in the fit is a free parameter of the algorithm: it is chosen by listing the fit terms according to their contribution to the $\chi^{2}$, and defining a threshold above which the contribution of further terms becomes negligible. The goodness of the fit has thus to be verified, as shown in Fig. A.1 for the application presented in the next section.

Once we have obtained the six equations relating the observables to the free parameters, the final stage of the algorithm is to solve this system of equations (system solving in Fig. 1). This task is carried out with the Mathematica software ${ }^{4}$, using the numerical FindInstance command, and the solutions have been reduced to the real domain. The computing time for this step depends strongly on the form of the equations in our system. In order to reduce it, we have fixed one of the parameters ( $\alpha_{1}$ for the application presented in the next section), and searched the solutions of the system for different given values of this frozen parameter. For each given value, we solve a system of five equations plus two inequalities, corresponding to the minimum and the maximum of $\Gamma_{\mathrm{GeV}^{5}}$. An additional inequality is added to the system, relating the size and the Doppler factor to the variability time-scale $\tau_{\text {var }}$,

$\tau_{\mathrm{var}} \geq \frac{1+z}{c} \frac{R}{\delta}$

where $c$ is the speed of light and $z$ is the redshift of the source.

To take into account the uncertainty on the five remaining observables, we iterate the solution of the system spanning the range $O_{i} \in\left[O_{i}-\sigma_{i}, O_{i}+\sigma_{i}\right]$ to produce a set of solutions. We sampled three different values of the frozen parameter, and we span the five observables 12 times each, leading to

http://root.cern.ch/

4 http://www.wolfram.com/mathematica/

5 There is no constraint on the choice of the frozen parameter ( $\alpha_{1}$ in this case), nor on the choice of the equation which is turned into two inequalities $\left(\Gamma_{\mathrm{GeV}}\right.$ in this case) to maintain the same number of equations and variables. 
Table 1. Summary of the constrained parameters for the SSC modelling of 1RXS J101015.9 - 311909.

\begin{tabular}{lccc}
\hline \hline$\alpha_{1}$ & 1.6 & 1.8 & 2.0 \\
\hline$\gamma_{\text {break }}$ & $(3.26-9.62) \times 10^{4}$ & $(3.43-11.21) \times 10^{4}$ & $(3.48-13.15) \times 10^{4}$ \\
$B[\mathrm{G}]$ & $(1.00-3.86) \times 10^{-2}$ & $(0.77-3.86) \times 10^{-2}$ & $(0.51-4.09) \times 10^{-2}$ \\
$K^{\prime}\left[\mathrm{cm}^{-3}\right]$ & $(0.061-14.70) \times 10^{-7}$ & $(0.023-9.92) \times 10^{-7}$ & $(0.0093-7.30) \times 10^{-7}$ \\
$\delta$ & $30.89-99.64$ & $30.76-99.91$ & $32.07-99.53$ \\
$R[\mathrm{~cm}]$ & $(0.43-8.28) \times 10^{16}$ & $(0.46-9.66) \times 10^{16}$ & $(0.49-11.57) \times 10^{16}$ \\
\hline
\end{tabular}

Notes. The solutions have been computed for three different values of $\alpha_{1}$, which have been frozen in the solution of the system. For the other five free parameters, we provide the minimum and the maximum values obtained, corresponding to the 1- $\sigma$ confidence interval. The solutions with a Doppler factor higher than 100 have been excluded. We note that the model parameters are correlated, as shown in the contour plots (Fig. 4).

$3 \times 12^{5}=746496$ systems studied. The Mathematica code has been parallelized as well, and run on a computer grid, using up to 200 cores simultaneously. For the expressions used for the application presented in the next section, the computing time is roughly nine hours.

The three steps of the analysis (grid production, grid fitting, and system solving, summarized in the plot shown in Fig. 1) can then take several hours, and this imposes a compromise between the resolution adopted for the grid (which affects the computing time of steps one and two), the number of terms used in the fit (which affects the computing time of steps two and three), and the number of iterations done in the system solution (which affects the computing time of step three).

The sets of parameters found with our algorithm are then used to produce contour plots, showing the confidence regions as a function of two different parameters, as in Fig. 4. As a final step, we compute all the SSC models corresponding to the found solutions, and we evaluate for each of them the reduced chi-square $\left(\tilde{\chi^{2}}\right)$ with respect to the observational data. This check allows us to estimate the true best-fit solution (the one with the minimum $\tilde{\chi^{2}}$ value), and to verify that the contour plots actually correspond to $1-\sigma$ contours (i.e. that all the solutions are characterised by $\Delta \tilde{\chi^{2}} \leq 2.3$ with respect to the best-fit value).

\section{Application: 1RXS J101015.9 - 311909}

In this section we present a first application of our numerical algorithm to the typical HBL 1RXS J101015.9 - 311909. The $\mathrm{TeV}$ emission from this source, located at a redshift of 0.143 (Piranomonte et al. 2007), has been detected with H.E.S.S. (Abramowski et al. 2012b). The multi-wavelength study presented in the H.E.S.S. discovery paper shows that there are two major systematic uncertainties in the SED of this object. The first one comes from a possible absorption effect in X-rays, affecting the evaluation of the position of the synchrotron peak; the second one comes from a problem in the determination of the Fermi spectrum at low energies due to a possible contamination by diffuse $\gamma$-ray emission from the Galactic foreground. In the following we consider that the synchrotron peak is located at an energy between the available UV and X-ray data (case B in Abramowski et al. 2012b), and the Fermi spectrum is evaluated using a $1 \mathrm{GeV}$ low-energy threshold. This does not represent a limitation of the algorithm, which can be successfully applied to the other cases discussed by Abramowski et al. (2012b) as well.

The value of $\alpha_{2}$ is uniquely constrained by the X-ray spectrum measured with Swift-XRT $\left(\Gamma_{X}=2.5 \pm 0.1\right)$ and it has been fixed at 4.0. The grid of simulated SEDs has been produced sampling the other six free parameters in the ranges: $\delta \in[20,100]$, $B \in[0.005,0.05] \mathrm{G}, \alpha_{1} \in[1.5,2.2], \gamma_{\text {break }} \in\left[3 \times 10^{4}, 1.3 \times 10^{5}\right]$,
$K^{\prime} \in\left[2 \times 10^{-9}, 2 \times 10^{-6}\right] \mathrm{cm}^{-3}$, and $R \in\left[4 \times 10^{15}, 10^{17}\right] \mathrm{cm}$. As laid out in the previous section, the grid has been produced sampling five different values for each parameter, logarithmically spaced (apart from $\alpha_{1}$ which has been sampled linearly) between the minimum and the maximum values. It is important to underline that solutions found outside of the sampled grid are not considered, because the system of equations is non-linear, and the fitted expression of the $O_{i}$ is appropriate only for the sampled parameter space. This could be seen as a limit of our approach, in the sense that we preselect a fraction of the parameter space. However, the analytical constraints defined by T98 allow us to beforehand exclude regions of the parameter space that are not accessible in the one-zone SSC model. In particular we decided not to study values of $\delta$ higher than 100 , as this would require extreme values of the bulk Lorentz factor of the emitting region.

The result of the parametrization of the six observables is

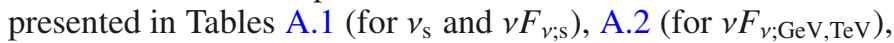
A.3 (for $\Gamma_{\mathrm{GeV}}$ ), and A.4 (for $\Gamma_{\mathrm{TeV}}$ ). As can be seen, while the expressions for $v_{\mathrm{s}}$ and $v f_{v ; \mathrm{s}}$ are simple, and consistent with what is expected from analytical considerations, the expressions for the Fermi and H.E.S.S. observables are more complicated, and we are obliged to consider second-order terms in the fitting polynomial with the extreme case of $\Gamma_{\mathrm{TeV}}$, which is described satisfactorily only when using more than one hundred terms. In Fig. A.1 we show the relation between the values of each observable, and the reconstructed values (after fit): in a perfect fit, the values should follow exactly the linear relation represented by the thick blue line.

The system of equations was then solved for three different values of $\alpha_{1}$ (equal to 1.6, 1.8, and 2.0) and for twelve different values of each observable (apart from $\Gamma_{\mathrm{GeV}}$, which is defined through two inequalities) reaching from $O_{i}-\sigma_{i}$ to $O_{i}+\sigma_{i}$. The range of values for $\alpha_{1}$ corresponds to expectations from standard acceleration scenarios. The values adopted for the observables are (in logarithm) $v_{\mathrm{s}} \in[16.15,16.25], v f_{v ; \mathrm{s}} \in[-10.74,-10.68]$, $v f_{v ; \mathrm{GeV}} \in[-11.98,-11.78], v f_{v ; \mathrm{TeV}} \in[-12.36,-12.10], \Gamma_{\mathrm{GeV}} \in$ $[-1.94,-1.48]$, and $\Gamma_{\mathrm{TeV}} \in[-3.55,-2.61]$. For the $\gamma$-ray observables, the considered error does include the systematic error on the observable (summed in quadrature to the statistic error). The system of equations includes an inequality for the variability time-scale $\left(\tau_{\mathrm{var}}=24 \mathrm{~h}\right)$ plus a limit on the value of the Doppler factor $(\delta<100$, which corresponds to the limit of the sampled grid). The results of our algorithm defining the range of SSC parameters which correctly fits the data are shown in Table 1 and the corresponding histograms are shown in Fig. 3.

To compare our solutions with the observational data, in Fig. 2 we show the SED of 1RXS J101015.9 - 311909, together with all the modelled SEDs found with our algorithm. The $\tilde{\chi^{2}}$ of 


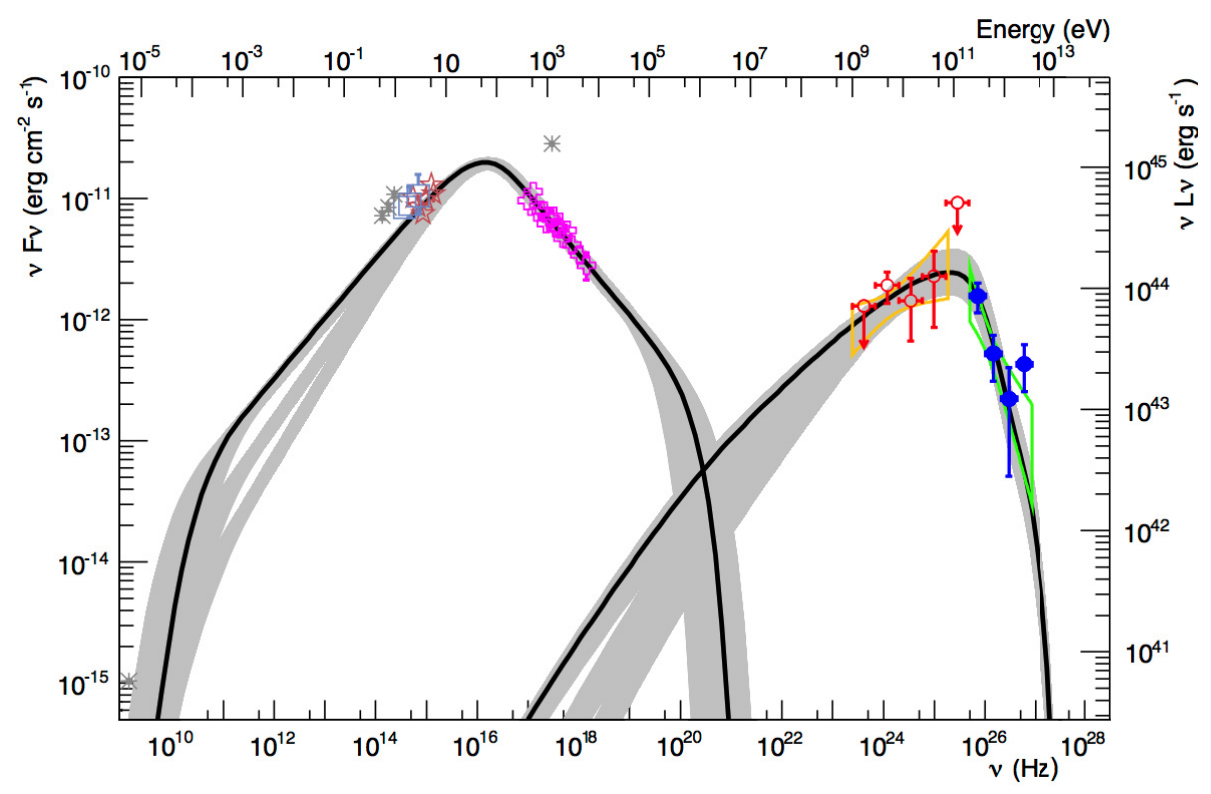

Fig. 2. SED of 1RXS J101015.9 - 311909 (Abramowski et al. 2012b, ; the H.E.S.S. spectrum is represented by the green bow-tie and the blue points, the Fermi-LAT spectrum by the orange bow-tie and the red empty circles; Swift-XRT data are shown by the pink crosses, Swift-UVOT data by the red stars, ATOM data by the blue open boxes, and archival data from the NED in grey). All the SSC models which describe the SED, as found with our algorithm, are plotted in grey, while the solid black curve represents the best-fit solution with $\tilde{\chi^{2}}=1.06$. It is characterised by an extreme value of $\delta=$ 96.83, $B=0.015 \mathrm{G}, R=1.3 \times 10^{16} \mathrm{~cm}, \alpha_{1}=$ $2.0, K^{\prime}=8.94 \times 10^{-8} \mathrm{~cm}^{-3}$, and $\gamma_{\mathrm{br}}=5.31 \times 10^{4}$. The three different families of solutions, which can be distinguished in the range between $10^{11}$ and $10^{14} \mathrm{~Hz}$, correspond to $\alpha_{1}=1.6,1.8$, and 2.0, as discussed in Sect. 3. The infrared and visible data can be reproduced by taking into account the host-galaxy contribution.

each modelled SED is estimated taking into account X-ray and $\gamma$-ray data only (i.e. excluding optical/UV and archival data), and the best-fit solution, shown in black in Fig. 2, has $\tilde{\chi^{2}}=1.06$.

In Fig. 4 we show the contour plots corresponding to our solutions for four different pairs of parameters: $B-\delta, R-\delta, \gamma_{\mathrm{br}^{-}}$ $B^{2} R$, and $u_{\mathrm{e}}-u_{B}$ (the particle and magnetic field energy densities). The different sets of solutions that can be seen in the contour plots are the result of the system of equations solved for discrete values of the observables in the range $\pm 1 \sigma$. The only contour which is statistically significant is the most extended one, that represents the $1-\sigma$ confidence region.

Our algorithm, unlike the standard modelling approach, permits us to provide general, quantitative constraints on the physical parameters of the emitting region. These are discussed in the following for the case of 1RXS J101015.9 - 311909.

\subsection{Doppler factor}

The first important result is that we can define a minimum Doppler factor that solves the system: $\delta_{\min } \approx 31$. In Fig. 4 (topleft plot) we show the contour plots of all our solutions in the $B-\delta$ plane, and we compare them to the analytical constraints computed following Tavecchio et al. (1998). It becomes clear that with our numerical approach we significantly narrow down the accessible parameter space.

The constraints on $\delta$ come mainly from the TeV slope and the variability time-scale; solutions with a lower Doppler factor exist, but would imply a variability time-scale higher than the one observed. In particular, comparing our result with the one presented by Abramowski et al. (2012b), the fact that they provide a solution with $\delta=30$ is due to a variability time-scale slightly higher than what has been assumed here $\left(\tau_{\text {var }} \approx 25 \mathrm{~h}\right)$ and to a rather soft $\mathrm{TeV}$ slope of the SSC model.

\subsection{Size of the emitting region}

The size of the emitting region is limited by the variability time scale (Eq. (4)), following the usual considerations in SSC modelling. The maximum allowed size is $\approx 10^{17} \mathrm{~cm}$ (at the edge of the values used to sample the grid). In Fig. 4 (top-right plot) we show the contour plots in the $R-\delta$ plane, and the limit corresponding to Eq. (4). As discussed above, solutions with a larger size of the emitting region and a lower Doppler factor exist, but violate the variability constraint. For the case of 1RXS J101015.9 - 311909, the variability constraint is relatively weak (no flaring behaviour observed), but for rapidly flaring sources this would permit us to constrain the parameter space even more.

\subsection{Energy budget}

An open question in blazar physics concerns the energy budget of the emitting region. For each solution we compute the magnetic energy density $\left(u_{B}=B^{2} / 8 \pi\right.$ in CGS units) and the particle energy density $\left(u_{\mathrm{e}}=m_{\mathrm{e}} c^{2} \int \mathrm{d} \gamma \gamma N(\gamma)\right)$. In Fig. 4 (bottom-right plot) all the solutions we found are shown in the $u_{\mathrm{e}}-u_{B}$ plane, indicating that the system is significantly out of equipartition, with a $u_{\mathrm{e}} / u_{B}$ ratio comprised between 10 and 200 . The evaluation of $u_{\mathrm{e}}$ depends on our assumptions on the low-energy part of the particle population (which is not constrained by the data), and in particular on the value of $\gamma_{\min }$. A higher value of $\gamma_{\min }$ would lower the value of $u_{\mathrm{e}}$, reducing the equipartition-ratio. This effect has not been tested here, because high values of $\gamma_{\min }$ affect the evaluation of the $\mathrm{GeV}$ slope, and would require a dedicated grid of SED model curves. However, for values of $\alpha_{1}$ lower than 2.0 , we do not expect that high values of $\gamma_{\min }$ affect significantly this result, while it should be important when $\alpha_{1} \geq 2.0$.

The fact that the SSC modelling of HBLs requires that the emitting region be out of equipartition is consistent with the results obtained by several authors (see e.g. Abdo et al. (2011b) for the case of Mrk 421, Abdo et al. (2011a) for the case of Mrk 501, and Abramowski et al. (2012a) for the case of PKS 2155-304).

\subsection{Particle energy distribution}

The study of the solutions for different values of $\alpha_{1}$ reveals that the slope of the electron distribution is not as well constrained by the GeV data as it is generally assumed to be (and as in T98). The solutions for the different $\alpha_{1}$ values are quite similar. An additional constraint on the values of the index of the particle population can be provided by optical/UV measurements. In the case of 1RXS J101015.9 - 311909, however, the low-energy data 

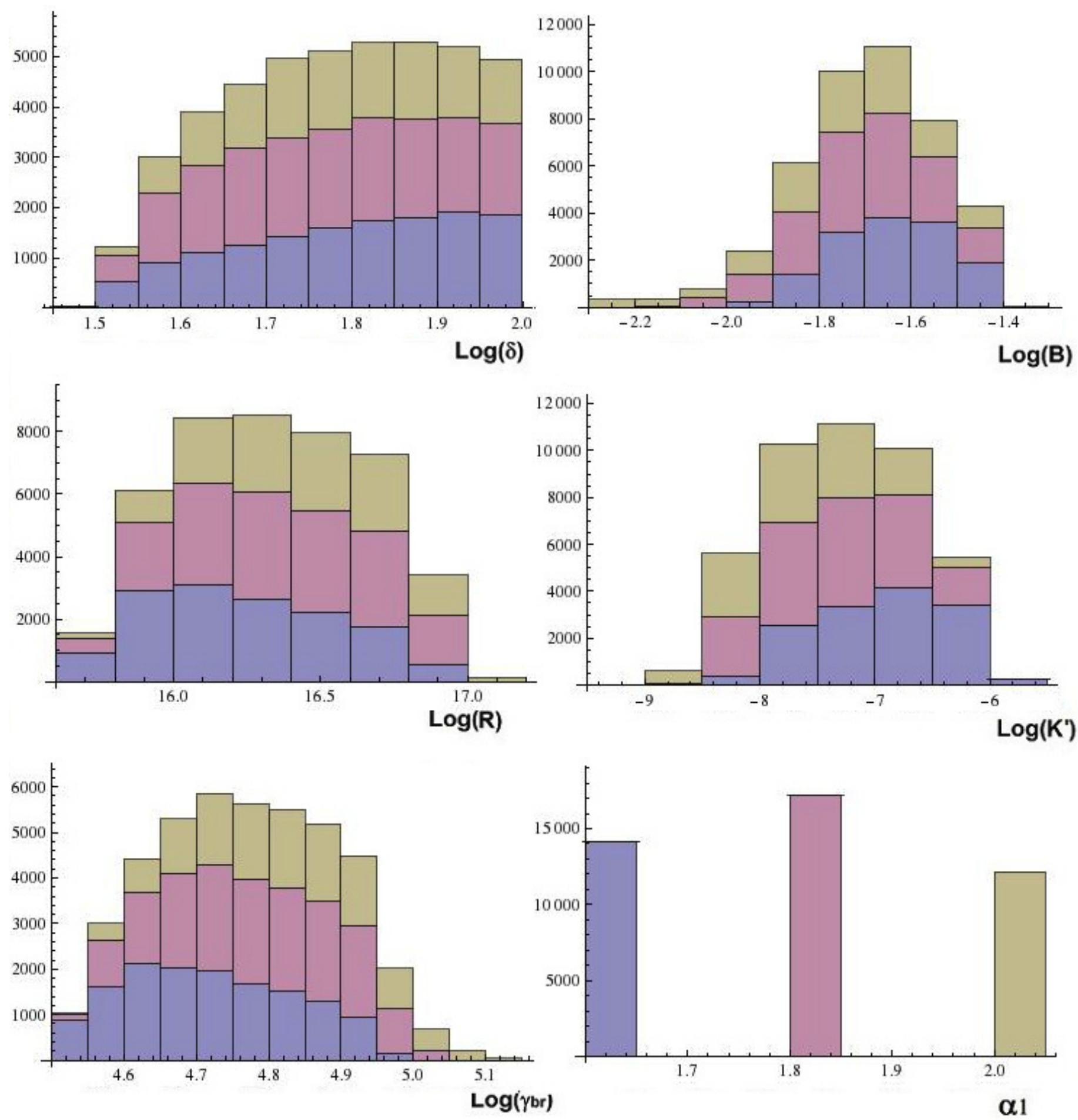

Fig. 3. Histograms showing the values of the SSC model parameters for the case of 1RXS J101015.9 - 311909. From top to bottom, and left to right, we show the distribution of the solutions for $\delta, B$ (in $\mathrm{G}), R$ (in $\mathrm{cm}$ ), $K^{\prime}$ (in $\mathrm{cm}^{-3}$ ), $\gamma_{\text {break }}$, and $\alpha_{1}$. We note that the value of $\alpha_{1}$ has been frozen and studied for three different cases $\left(1.6,1.8\right.$, and 2.0). The three colours represent the different solutions for the three values of $\alpha_{1}$ studied: violet-1.6, pink-1.8, and yellow-2.0.

cannot be used to define a unique $\alpha_{1}$ value because of the uncertainty on the correction by absorption and by the host-galaxy contamination (see Abramowski et al. 2012b, and Fig. 2). For this source we can use the optical and UV data only as upper limits, excluding slopes softer than 2.2. For other blazars, with a better estimate of the AGN emission in this part of the spectrum, a more precise value of $\alpha_{1}$ can be determined.
The values of $\alpha_{1}$ that we have considered point to one of the open questions of blazar physics: synchrotron cooling predicts a spectral break equal to one (i.e. $\alpha_{1}=3.0$, for $\alpha_{2}=4.0$, as constrained by the X-ray observations), which is clearly excluded by the data. This might indicate that the homogeneous one-zone model is too simple, and that more complex effects should be taken into account, such as non-linear inverse Compton cooling, 
M. Cerruti et al.: Constraining the parameter space of the one-zone SSC model for GeV-TeV detected BL Lacertae objects

B- $\delta$

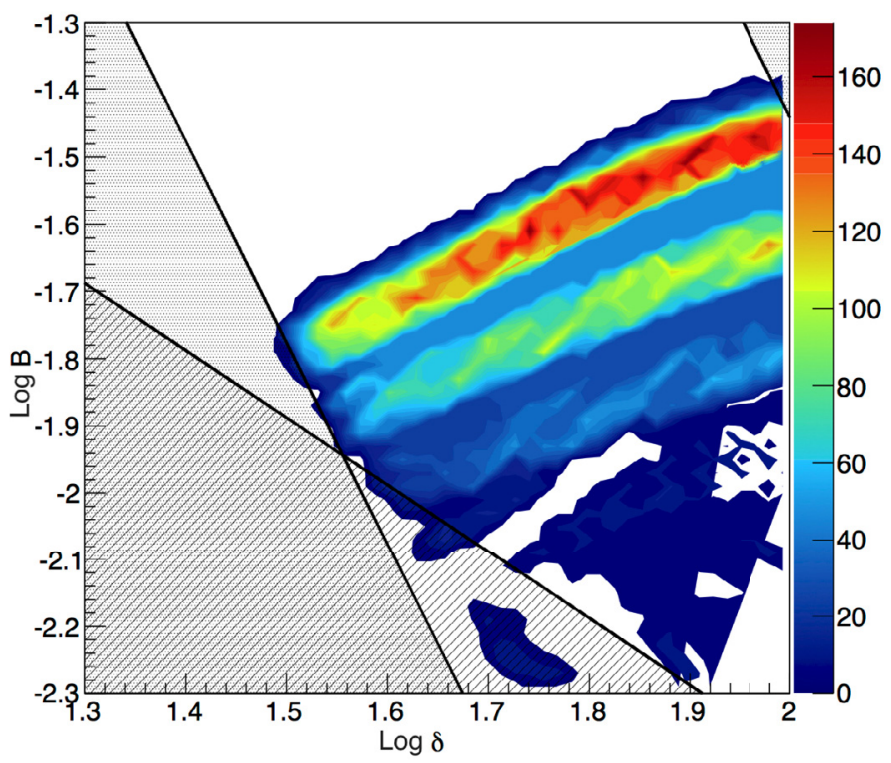

$\gamma_{b r}$

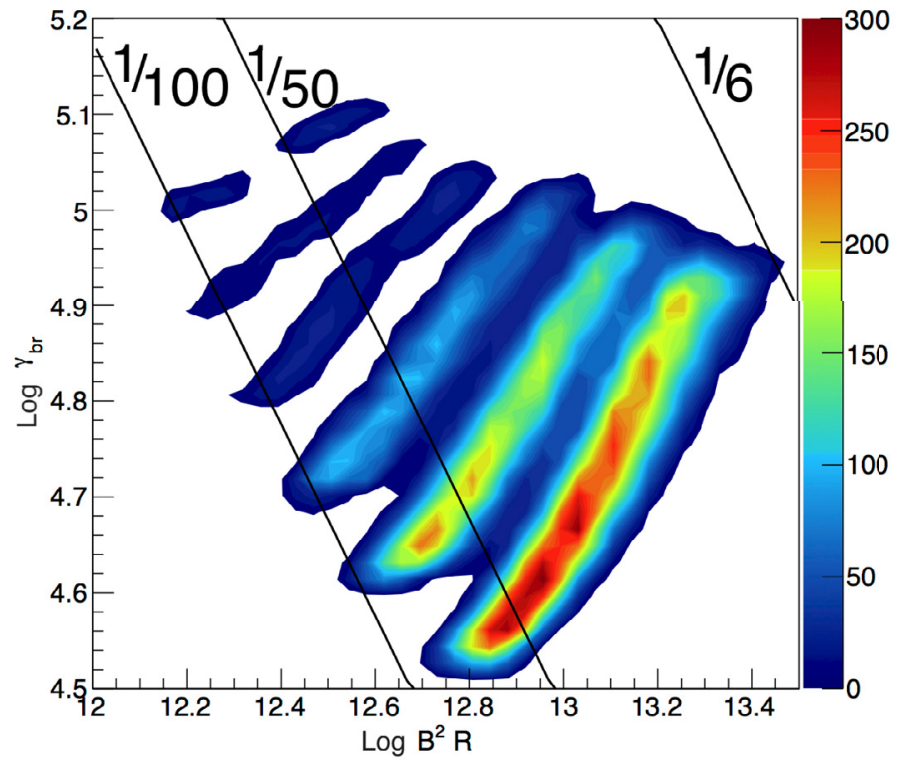

R- $\delta$

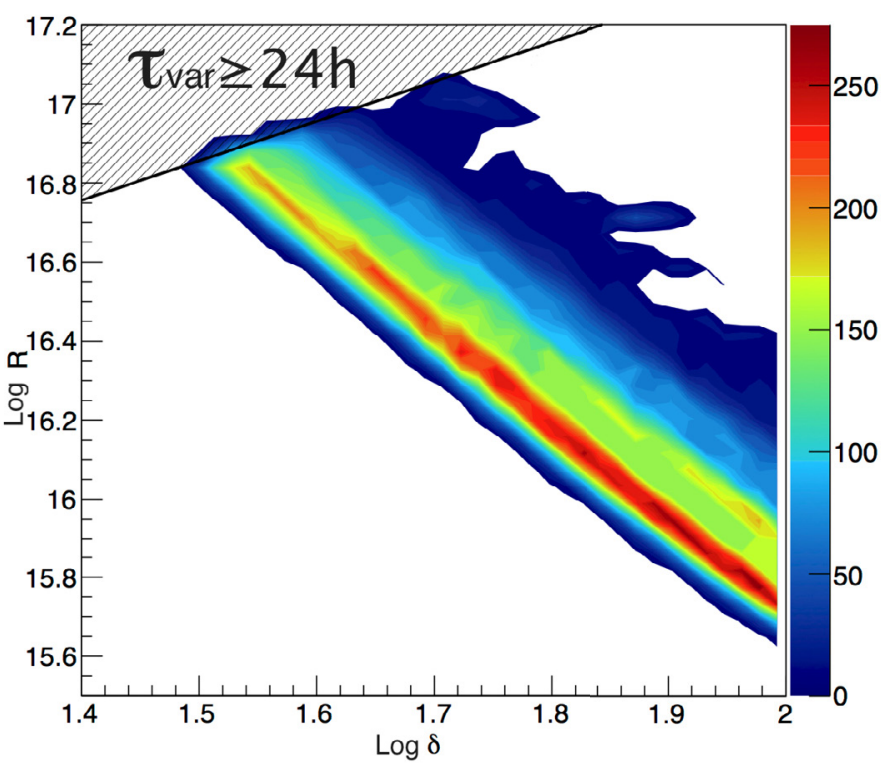

Energy densities

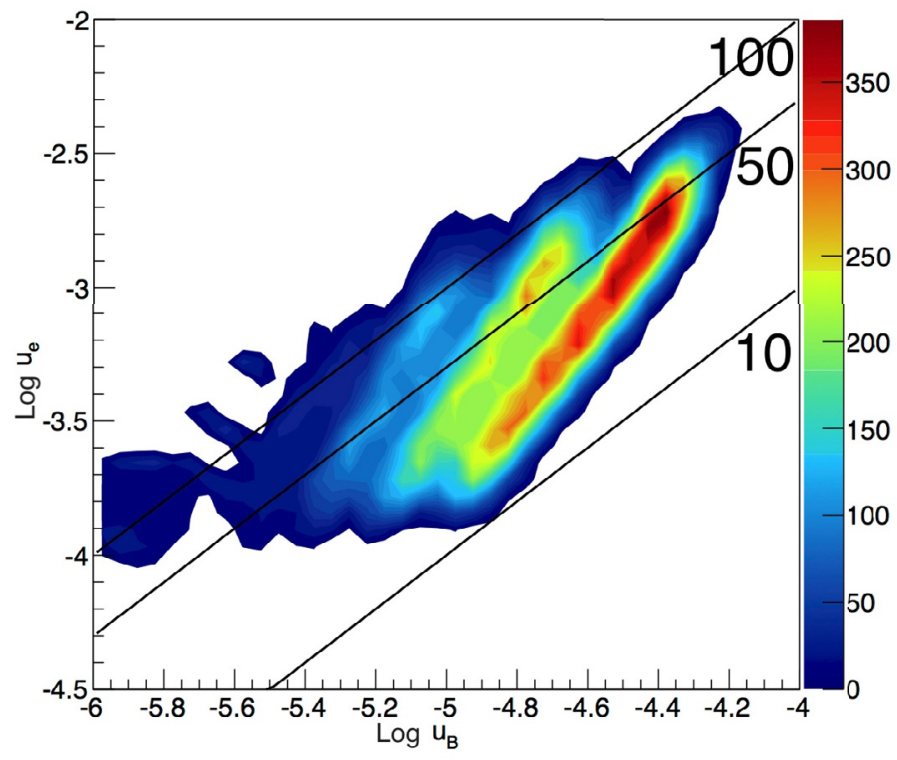

Fig. 4. Contour plots of the solutions found for 1RXS J101015.9 - 311909. The contours are expressed in arbitrary units representing the number of solutions corresponding to a given pair of parameters. White represents zero. As discussed in Sect. 2, the most extended contour corresponds to a 1- $\sigma$ contour with respect to the best-fit solution, as determined a posteriori. Top left: contours in the $B-\delta$ plane. The shadowed regions represent the exclusion regions defined following T98 (the region filled with diagonal lines is computed from Eq. (4), while the region filled with dots is computed from Eq. (11), considering an emitting region size equal to $(1+z) c \tau_{\text {var }} / \delta$ and $\left.(1+z) c \tau_{\text {var }} / 30 \delta\right)$. We do not include the constraint on the consistency of $\gamma_{\text {break }}$ with synchrotron cooling). Top right: contours in the $R-\delta$ plane. The filled region corresponds to a variability time-scale higher than 24 h. Bottom left: contours in the $\gamma_{\text {break }}-B^{2} R$ plane. The black lines represent the expected value of $\gamma_{\text {break }}$ in the presence of pure synchrotron cooling, assuming a value of $\beta_{\text {esc }}$ equal to (from left to right) $1 / 100,1 / 50$, and $1 / 6$. Bottom right: contours in the $u_{\mathrm{e}}-u_{B}$ plane. The black lines correspond to $u_{\mathrm{e}} / u_{B}$ values equal to (from bottom to top) 10 , 50 , and 100 , respectively.

energy-dependent acceleration and escape from the emitting region, non-homogeneity of both particles, and magnetic field. This justifies the choice of considering the two particle slopes $\alpha_{1}$ and $\alpha_{2}$ as independent parameters.

If the spectral break were a result of pure synchrotron cooling, the energy of the break in the particle distribution could be computed theoretically, and linked to the other free parameters. Assuming that particles are injected in the emitting region, escape from it at a speed $\beta_{\mathrm{esc}} c^{6}$, and lose their energy while emitting synchrotron radiation, the value of $\gamma_{\text {break }}$ can be expressed as (see T98, Eq. (30))

$\gamma_{\text {break }}=\beta_{\mathrm{esc}} c \frac{5 \times 10^{8}}{B^{2} R}$.

6 The particle escape term can be considered as well as an adiabatic term (i.e. associated to the expansion of the emitting region). 
In Fig. 4 (bottom-right plot) we show the values of $\gamma_{\text {break }}$ that we found, as a function of $B^{2} R$, comparing them to the theoretical expectations. As can be seen, our solutions are consistent with a pure synchrotron cooling only if the escape speed is lower than $c / 6$. This value can be increased by considering additional cooling terms, such as the energy loss by inverse Compton scattering. This point strengthens the fact that the observed spectral break is not consistent with pure synchrotron cooling, and that more complex effects have to be considered to explain the particle spectrum in the stationary state.

In this application we have fixed the values of $\gamma_{\min }$ and $\gamma_{\text {Max }}$, which cannot be constrained in the particular case of 1RXS J101015.9 - 311909. For other sources with different observational constraints it would be possible to fix other parameters (such as $\alpha_{1}$, in the presence of precise measurements at lower frequencies) and to study $\gamma_{\min }$ or $\gamma_{\operatorname{Max}}$. In particular, this approach would be interesting for some other HBLs, for which a value of $\gamma_{\min }$ higher than the one that has been assumed here is required in order to describe the SED with a SSC model (see e.g. Katarzyński et al. 2006; Kaufmann et al. 2011).

\section{Conclusions}

In this paper we have described a new algorithm to constrain the parameter space of the one-zone SSC model, that can be successfully applied to any HBL with simultaneous spectral measurements in the $\mathrm{X}$-ray, $\mathrm{GeV}$, and $\mathrm{TeV}$ energy-range. The algorithm follows the idea developed by T98 (i.e. the definition of a set of equations linking the free model parameters and the blazar observables), but is fully numerical, introduces as new observables the properties of the $\mathrm{GeV}$ and the $\mathrm{TeV}$ detection, and allows the derivation of a new set of equations.

The algorithm cannot be applied to the subclasses of LBLs and FSRQs because for these sources an external inverse Compton component is required, leading to a higher number of free parameters in the model.

As a first test, we have applied our algorithm to the typical HBL 1RXS J101015.9 - 311909 and derived the set of solutions that correctly describes its SED. It is important to underline that the range of solutions found here is much narrower than the one obtained with the analytical approach, showing that the information coming from the $\mathrm{GeV}-\mathrm{TeV}$ detection permits the model to be better constrained. In this way, a true best-fit solution is provided.

Other numerical algorithms have been proposed in the literature to constrain the SSC model parameter space. Both Finke et al. (2008) and Mankuzhiyil et al. (2011) have proposed a $\chi^{2}$ minimization algorithm to fit the SEDs of BL Lac objects, showing as well that, thanks to the $\mathrm{TeV}$ detection, it is possible to obtain a best-fit solution. Neither of these two methods make use, however, of the recent Fermi data. On the other hand, their fits are strongly constrained by the low energy (optical and UV) photons, implicitly assuming that the $\gamma$-ray emitting region is responsible for the totality of this flux. Our method, based on the properties of the Fermi detection and the position of the synchrotron peak, allows us to relax this hypothesis, and to model more complex scenarios in which the low-energy flux is contaminated by emission from other, farther regions of the jet, or the host galaxy. It is worth noting as well that the method described by Finke et al. (2008) introduces the requirement that the energy budget of the emitting region is minimized, and pre-selects a set of solutions.

Several improvements to the algorithm we have presented here can be considered. First of all, the production of the grid of modelled SEDs, as was discussed here for the application to 1RXS J101015.9 - 311909, limits the parameter space: a larger grid can be produced, extending the region of the parameter space under investigation. To reduce the computing time we have also frozen the value of $\alpha_{1}$, solving the system for different, given values of this parameter; however, the system can, in principle, be solved leaving all the parameters free to vary, studying $\alpha_{1}$ as a free parameter as well.

In most of the current publications, the standard approach in blazar physics is still to consider the one-zone SSC model as degenerate (i.e. the number of free parameters is higher than the number of constraints), and a fit of the SED is usually not performed. It is thus common to explore only a few solutions to decide whether the data can be described in a satisfactory way, without studying the entire parameter space nor evaluating the errors on the parameters. We have shown here that, at least for GeV-TeV HBLs, it is possible to explore the parameter space more systematically and to significantly improve the constraints on the parameters of the model. Our algorithm can be applied to the continuously increasing sample of HBLs detected in $\gamma$-rays, and will help us improve our comprehension of this extreme class of blazars.

Acknowledgements. The work has been performed using the computation facilities of the Paris Observatory and the Harvard-Smithsonian Center for Astrophysics. The authors wish to thank Hélène Sol for fruitful discussions, as well as the anonymous referee for the comments and remarks which improved the present work.

\section{References}

Abdo, A. A., Ackermann, M., Agudo, I., et al. 2010, ApJ, 716, 30 Abdo, A. A., Ackermann, M., Ajello, M., et al. 2011a, ApJ, 727, 129 Abdo, A. A., Ackermann, M., Ajello, M., et al. 2011b, ApJ, 736, 131 Abramowski, A., Acero, F., Aharonian, F., et al. 2012a, A\&A, 539, A149

Abramowski, A., Acero, F., et al. (H.E.S.S. Collaboration) 2012b, A\&A, 542, A94

Aharonian, F. A., Khangulyan, D., \& Costamante, L. 2008, MNRAS, 387, 1206 Angel, J. R. P., \& Stockman, H. S. 1980, ARA\&A, 18, 321

Atwood, W. B., Abdo, A. A., Ackermann, M., et al. 2009, ApJ, 697, 1071

Dermer, C. D., \& Schlickeiser, R. 1993, ApJ, 416, 458

Dermer, C. D., Schlickeiser, R., \& Mastichiadis, A. 1992, A\&A, 256, L27

Finke, J. D., Dermer, C. D., \& Böttcher, M. 2008, ApJ, 686, 181

Fossati, G., Maraschi, L., Celotti, A., Comastri, A., \& Ghisellini, G. 1998 , MNRAS, 299, 433

Franceschini, A., Rodighiero, G., \& Vaccari, M. 2008, A\&A, 487, 837

Ghisellini, G., Tavecchio, F., Foschini, L., \& Ghirlanda, G. 2011, MNRAS, 414 2674

Inoue, S., \& Takahara, F. 1996, ApJ, 463, 555

Katarzyński, K., Sol, H., \& Kus, A. 2001, A\&A, 367, 809

Katarzyński, K., Ghisellini, G., Tavecchio, F., Gracia, J., \& Maraschi, L. 2006, MNRAS, 368, L52

Kaufmann, S., Wagner, S. J., Tibolla, O., \& Hauser, M. 2011, A\&A, 534, A130 Konigl, A. 1981, ApJ, 243, 700

Mankuzhiyil, N., Ansoldi, S., Persic, M., \& Tavecchio, F. 2011, ApJ, 733, 14

Mannheim, K. 1993, A\&A, 269, 67

Moore, R. L., \& Stockman, H. S. 1981, ApJ, 243, 60

Mücke, A., \& Protheroe, R. J. 2001, APh, 15, 12

Nieppola, E., Valtaoja, E., Tornikoski, M., Hovatta, T., \& Kotiranta, M. 2008, A\&A, 488, 867

Padovani, P., \& Giommi, P. 1995, ApJ, 444, 567

Padovani, P., Giommi, P., \& Rau, A. 2012, MNRAS, L423

Piranomonte, S., Perri, M., Giommi, P., Landt, H., \& Padovani, P. 2007, A\&A, 470, 787

Salamon, M. H., \& Stecker, F. W. 1998, ApJ, 493, 547

Sikora, M., Begelman, M. C., \& Rees, M. J. 1994, ApJ, 421, 153

Stein, W. A., Odell, S. L., \& Strittmatter, P. A. 1976, ARA\&A, 14, 173

Tavecchio, F., Maraschi, L., \& Ghisellini, G. 1998, ApJ, 509, 608

Urry, C. M., \& Padovani, P. 1995, PASP, 107, 803 


\section{Appendix A: Tables and figures containing the fit results}

We present here the results of the fitting of the grid for the particular case of 1RXS J101015.9 - 311909. For each observable we provide the terms of the fit listed by increasing polynomial order of the parameters composing each term. The plot showing the goodness of each fit (comparison between the sampled and the reconstructed values) are given in Fig. A.1.
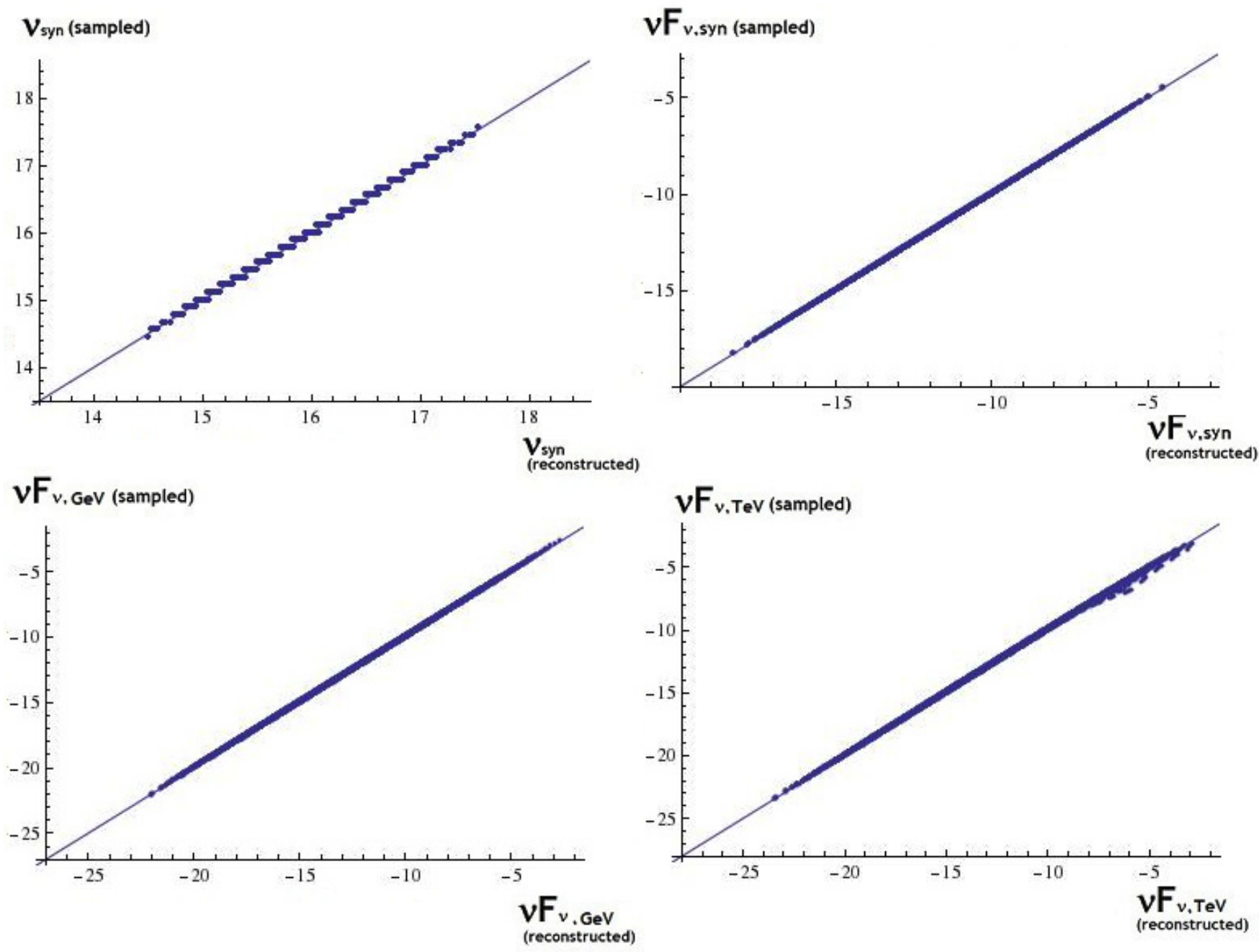

\section{$\Gamma_{\mathrm{GeV} \text { (sampled) }}$}
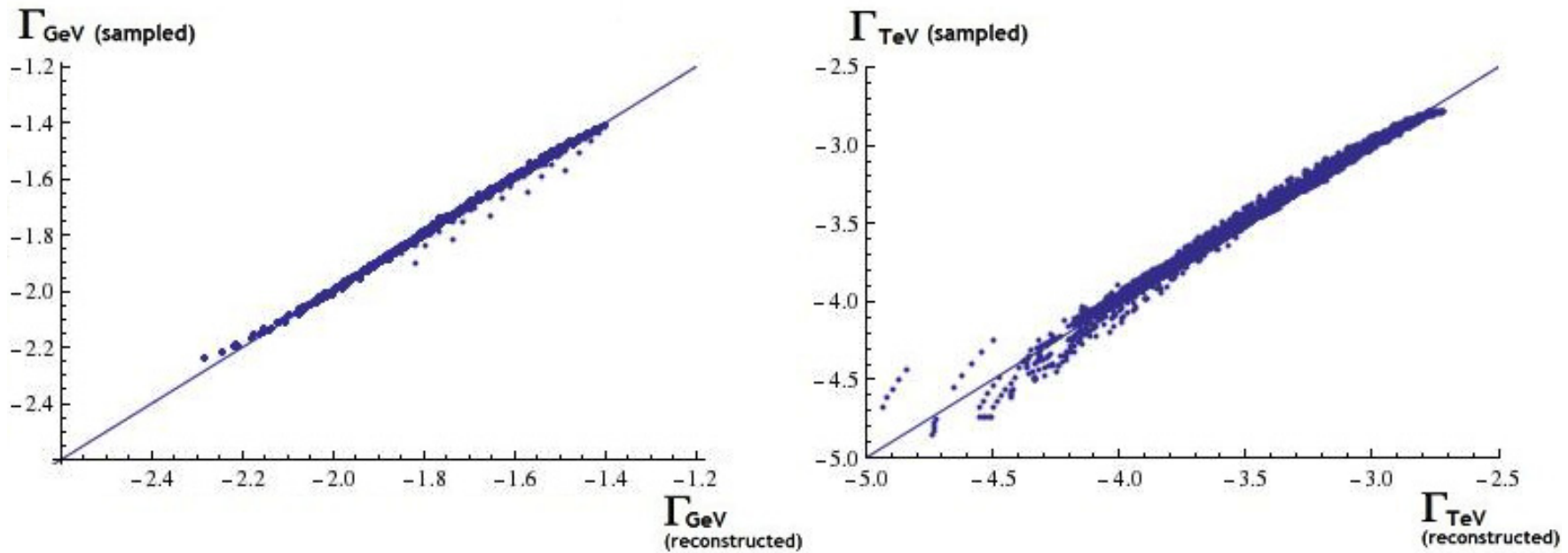

Fig. A.1. Comparison between the sampled values of the six observables considered in this study and their reconstructed values, expressed as a function of the SSC-model parameters, for the case of 1RXS J101015.9 - 311909. In a perfect fit, the points would follow a linear relation (thin solid line). The six subplots are in the order (from top to bottom, and left to right), the synchrotron peak frequency $\left(v_{\mathrm{s}}\right)$, the synchrotron peak flux (expressed as $v F_{v ; \mathrm{s}}$ ), the Fermi flux (measured at the decorrelation energy and expressed as $v F_{v ; \mathrm{GeV}}$ ), the H.E.S.S. flux (measured at the decorrelation energy and expressed as $\left.v F_{v ; \mathrm{TeV}}\right)$, the measured Fermi photon index $\left(\Gamma_{\mathrm{GeV}}\right)$, and the measured H.E.S.S. photon index $\left(\Gamma_{\mathrm{TeV}}\right)$. 
A\&A 558, A47 (2013)

Table A.1. Coefficients of the fit performed to obtain an expression of $v_{\mathrm{s}}(l e f t)$ and $v F_{v ; \mathrm{s}}(r i g h t)$ for the case of 1RXS J101015.9 - 311909.

\begin{tabular}{lcccccccccccccccc}
\hline \hline & \multicolumn{1}{c}{$v_{\mathrm{s}}$} & \multicolumn{11}{c}{$v F_{\gamma ; \mathrm{s}}$} \\
\hline Order & $\gamma_{\mathrm{br}}$ & $B$ & $K^{\prime}$ & $\alpha_{1}$ & $\delta$ & $R$ & Coefficient & Order & $\gamma_{\mathrm{br}}$ & $B$ & $K^{\prime}$ & $\alpha_{1}$ & $\delta$ & $R$ & Coefficient \\
\hline 0 & - & - & - & - & - & - & 6.81783 & 0 & - & - & - & - & - & - & -70.5346 \\
1 & 1 & - & - & - & - & - & 1.99229 & 1 & 1 & - & - & - & - & - & 2.99479 \\
1 & - & 1 & - & - & - & - & 0.999460 & 1 & - & 1 & - & - & - & - & 1.99998 \\
1 & - & - & - & 1 & - & - & -0.852880 & 1 & - & - & 1 & - & - & - & 1.00000 \\
1 & - & - & - & - & 1 & - & 1.00159 & 1 & - & - & - & 1 & - & - & 0.220701 \\
& & & & & & & & & 1 & - & - & - & - & 1 & - & 3.99998 \\
& & & & & & & & 1 & - & - & - & - & - & 1 & 3.00004 \\
\hline
\end{tabular}

Notes. The corresponding plots are the top plots of Fig. A.1. Each line corresponds to a term of the fit: the first column gives the total order of the polynomial, the next six columns give the degree considered for each model free parameter, and the last column gives the associated coefficient. The terms are listed with respect to the parameters composing each term, and then by increasing degree, not by relative weight in the fit. In this case, the value of $\alpha_{2}$ has been fixed to 4.0.

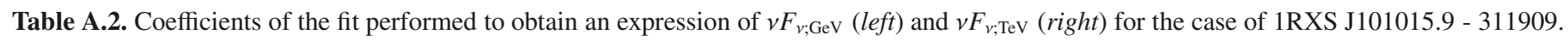

\begin{tabular}{lcccccccccccccccc}
\hline \hline \multicolumn{1}{c}{$v F_{v ; \mathrm{GeV}}$} & \multicolumn{1}{c}{$v F_{v ; \mathrm{TeV}}$} \\
\hline Order & $\gamma_{\mathrm{br}}$ & $B$ & $K^{\prime}$ & $\alpha_{1}$ & $\delta$ & $R$ & Coefficient & Order & $\gamma_{\mathrm{br}}$ & $B$ & $K^{\prime}$ & $\alpha_{1}$ & $\delta$ & $R$ & Coefficient \\
\hline 0 & - & - & - & - & - & - & -115.648 & 0 & - & - & - & - & - & - & -114.123 \\
1 & 1 & - & - & - & - & - & 17.7128 & 1 & 1 & - & - & - & - & - & 13.7218 \\
2 & 2 & - & - & - & - & - & -1.58660 & 2 & 2 & - & - & - & - & - & -0.850289 \\
1 & - & 1 & - & - & - & - & 5.19307 & 1 & - & 1 & - & - & - & - & -4.40501 \\
2 & 1 & 1 & - & - & - & - & -0.813593 & 2 & 1 & 1 & - & - & - & - & 3.44968 \\
1 & - & - & 1 & - & - & - & 1.99993 & 3 & 2 & 1 & - & - & - & - & -0.444764 \\
1 & - & - & - & 1 & - & - & 16.9990 & 1 & - & - & 1 & - & - & - & 1.99314 \\
2 & 1 & - & - & 1 & - & - & -14.1166 & 1 & - & - & - & 1 & - & - & -10.8581 \\
3 & 2 & - & - & 1 & - & - & 2.34452 & 2 & 1 & - & - & 1 & - & - & 2.34036 \\
2 & - & 1 & - & 1 & - & - & -4.22521 & 3 & - & - & - & 3 & - & - & 2.69285 \\
3 & 1 & 1 & - & 1 & - & - & 1.19513 & 1 & - & - & - & - & 1 & - & -13.1497 \\
1 & - & - & - & - & 1 & - & 5.74872 & 2 & 1 & - & - & - & 1 & - & 8.23156 \\
2 & 1 & - & - & - & 1 & - & -0.506995 & 3 & 2 & - & - & - & 1 & - & -0.942506 \\
2 & - & - & - & 1 & 1 & - & 1.59946 & 1 & - & - & - & - & - & 1 & 3.98568 \\
1 & - & - & - & - & - & 1 & 3.99993 & & & & & & & \\
\hline
\end{tabular}

Notes. The corresponding plots are the central plots of Fig. A.1. For more details see the caption of Table A.1.

Table A.3. Coefficients of the fit performed to obtain an expression of $\Gamma_{\mathrm{GeV}}$ for the case of 1RXS J101015.9 - 311909 .

\begin{tabular}{lcccccccccccccccc}
\hline \hline \multicolumn{10}{c}{$\Gamma_{\mathrm{GeV}}$} \\
\hline Order & $\gamma_{\mathrm{br}}$ & $B$ & $K^{\prime}$ & $\alpha_{1}$ & $\delta$ & $R$ & Coefficient & Order & $\gamma_{\mathrm{br}}$ & $B$ & $K^{\prime}$ & $\alpha_{1}$ & $\delta$ & $R$ & Coefficient \\
\hline 0 & - & - & - & - & - & - & 215.795 & 2 & 1 & - & - & 1 & - & - & -16.2468 \\
1 & 1 & - & - & - & - & - & -202.197 & 3 & 2 & - & - & 1 & - & - & 1.54404 \\
2 & 2 & - & - & - & - & - & 67.9303 & 2 & - & 1 & - & 1 & - & - & -0.383771 \\
3 & 3 & - & - & - & - & - & -9.87975 & 2 & - & - & - & 2 & - & - & -2.36309 \\
4 & 4 & - & - & - & - & - & 0.528434 & 1 & - & - & - & - & 1 & - & -59.7992 \\
1 & - & 1 & - & - & - & - & -53.6426 & 2 & 1 & - & - & - & 1 & - & 39.7326 \\
2 & 1 & 1 & - & - & - & - & 35.3010 & 3 & 2 & - & - & - & 1 & - & -8.64464 \\
3 & 2 & 1 & - & - & - & - & -7.62267 & 4 & 3 & - & - & - & 1 & - & 0.621649 \\
4 & 3 & 1 & - & - & - & - & 0.542311 & 3 & 1 & 1 & - & - & 1 & - & -0.0933089 \\
3 & 1 & 2 & - & - & - & - & -0.0421037 & 4 & 2 & 1 & - & - & 1 & - & 0.017273 \\
4 & 2 & 2 & - & - & - & - & 0.00702904 & 2 & - & - & - & 1 & 1 & - & -0.293701 \\
1 & - & - & - & 1 & - & - & 41.7990 & 2 & - & - & - & - & 2 & - & -0.0945532 \\
\hline
\end{tabular}

Notes. The corresponding plot is the bottom-left plot of Fig. A.1. For more details see the caption of Table A.1. 
M. Cerruti et al.: Constraining the parameter space of the one-zone SSC model for GeV-TeV detected BL Lacertae objects

Table A.4. Coefficients of the fit performed to obtain an expression of $\Gamma_{\mathrm{TeV}}$ for the case of 1RXS J101015.9 - 311909.

\begin{tabular}{|c|c|c|c|c|c|c|c|c|c|c|c|c|c|c|c|}
\hline \multicolumn{16}{|c|}{$\Gamma_{\mathrm{TeV}}$} \\
\hline Order & $\gamma_{\mathrm{br}}$ & $B$ & $K^{\prime}$ & $\alpha_{1}$ & $\delta$ & $R$ & Coefficient & Order & $\gamma_{\mathrm{br}}$ & $B$ & $K^{\prime}$ & $\alpha_{1}$ & $\delta$ & $R$ & Coefficient \\
\hline 0 & - & - & - & - & - & - & 433.763 & 3 & - & 1 & - & 1 & 1 & - & -0.688389 \\
\hline 1 & 1 & - & - & - & - & - & 1660.83 & 2 & - & - & - & - & 2 & - & -53.2164 \\
\hline 2 & 2 & - & - & - & - & - & -536.572 & 4 & 2 & - & - & - & 2 & - & 5.94909 \\
\hline 3 & 3 & - & - & - & - & - & 17.0195 & 5 & 3 & - & - & - & 2 & - & -0.738527 \\
\hline 1 & - & 1 & - & - & - & - & 872.651 & 4 & 1 & 1 & - & - & 2 & - & 0.943224 \\
\hline 2 & 1 & 1 & - & - & - & - & -83.0061 & 5 & 2 & 1 & - & - & 2 & - & -0.195947 \\
\hline 3 & 2 & 1 & - & - & - & - & -83.7787 & 3 & - & - & - & - & 3 & - & 7.96991 \\
\hline 4 & 3 & 1 & - & - & - & - & 5.13618 & 4 & 1 & - & - & - & 3 & - & -1.73813 \\
\hline 5 & 4 & 1 & - & - & - & - & 0.120479 & 1 & - & - & - & - & - & 1 & -254.494 \\
\hline 2 & - & 2 & - & - & - & - & -209.046 & 2 & 1 & - & - & - & - & 1 & -102.434 \\
\hline 3 & 1 & 2 & - & - & - & - & 112.983 & 3 & 2 & - & - & - & - & 1 & 44.4274 \\
\hline 4 & 2 & 2 & - & - & - & - & -18.0429 & 2 & - & 1 & - & - & - & 1 & -213.596 \\
\hline 5 & 3 & 2 & - & - & - & - & 1.22248 & 3 & 1 & 1 & - & - & - & 1 & 63.8761 \\
\hline 1 & - & - & 1 & - & - & - & 937.346 & 4 & 2 & 1 & - & - & - & 1 & 1.03778 \\
\hline 2 & 1 & - & 1 & - & - & - & -215.023 & 3 & - & 2 & - & - & - & 1 & 4.71668 \\
\hline 3 & 2 & - & 1 & - & - & - & -17.4660 & 4 & 1 & 2 & - & - & - & 1 & -1.68036 \\
\hline 2 & - & 1 & 1 & - & - & - & 50.7081 & 2 & - & - & 1 & - & - & 1 & -122.306 \\
\hline 3 & 1 & 1 & 1 & - & - & - & -64.8202 & 3 & 1 & - & 1 & - & - & 1 & 21.0306 \\
\hline 4 & 2 & 1 & 1 & - & - & - & 2.94899 & 4 & 2 & - & 1 & - & - & 1 & 3.83924 \\
\hline 3 & - & 2 & 1 & - & - & - & 4.10333 & 3 & - & 1 & 1 & - & - & 1 & -13.7376 \\
\hline 4 & 1 & 2 & 1 & - & - & - & 2.54005 & 4 & 1 & 1 & 1 & - & - & 1 & 9.62879 \\
\hline 2 & - & - & 2 & - & - & - & 43.3093 & 5 & 2 & 1 & 1 & - & - & 1 & -0.221533 \\
\hline 3 & 1 & - & 2 & - & - & - & -21.8910 & 4 & - & 2 & 1 & - & - & 1 & -0.296910 \\
\hline 4 & 2 & - & 2 & - & - & - & 1.38984 & 5 & 1 & 2 & 1 & - & - & 1 & -0.197426 \\
\hline 3 & - & 1 & 2 & - & - & - & -25.7945 & 3 & - & - & 2 & - & - & 1 & -7.67172 \\
\hline 4 & 1 & 1 & 2 & - & - & - & 2.88766 & 4 & 1 & - & 2 & - & - & 1 & 2.90132 \\
\hline 5 & 2 & 1 & 2 & - & - & - & -0.0347576 & 5 & 2 & - & 2 & - & - & 1 & -0.0925614 \\
\hline 4 & - & 2 & 2 & - & - & - & 0.954052 & 4 & - & 1 & 2 & - & - & 1 & 2.60294 \\
\hline 5 & 1 & 2 & 2 & - & - & - & -0.0382109 & 5 & 1 & 1 & 2 & - & - & 1 & -0.170639 \\
\hline 3 & - & - & 3 & - & - & - & -2.10358 & 5 & - & 2 & 2 & - & - & 1 & -0.0489805 \\
\hline 4 & 1 & - & 3 & - & - & - & 0.471742 & 4 & - & - & 3 & - & - & 1 & 0.133559 \\
\hline 1 & - & - & - & 1 & - & - & -168.566 & 5 & 1 & - & 3 & - & - & 1 & -0.0299422 \\
\hline 2 & 1 & - & - & 1 & - & - & 89.7793 & 2 & - & - & - & - & 1 & 1 & -2.83619 \\
\hline 3 & 2 & - & - & 1 & - & - & -14.9943 & 3 & 1 & - & - & - & 1 & 1 & 0.931345 \\
\hline 4 & 3 & - & - & 1 & - & - & 0.783183 & 3 & - & 1 & - & - & 1 & 1 & 0.692465 \\
\hline 2 & - & 1 & - & 1 & - & - & 4.06062 & 3 & - & - & 1 & - & 1 & 1 & -0.341208 \\
\hline 3 & 1 & 1 & - & 1 & - & - & -0.0229217 & 4 & 1 & - & 1 & - & 1 & 1 & 0.112470 \\
\hline 4 & 2 & 1 & - & 1 & - & - & -0.132376 & 4 & - & 1 & 1 & - & 1 & 1 & 0.0839418 \\
\hline 1 & - & - & - & - & 1 & - & 449.564 & 2 & - & - & - & - & - & 2 & 6.46489 \\
\hline 2 & 1 & - & - & - & 1 & - & -44.9238 & 3 & 1 & - & - & - & - & 2 & 4.72897 \\
\hline 3 & 2 & - & - & - & 1 & - & -76.3805 & 4 & 2 & - & - & - & - & 2 & -1.66802 \\
\hline 4 & 3 & - & - & - & 1 & - & 21.1307 & 3 & - & 1 & - & - & - & 2 & 7.24229 \\
\hline 5 & 4 & - & - & - & 1 & - & -1.57986 & 4 & 1 & 1 & - & - & - & 2 & -1.92839 \\
\hline 2 & - & 1 & - & - & 1 & - & 251.194 & 5 & 2 & 1 & - & - & - & 2 & -0.0907203 \\
\hline 3 & 1 & 1 & - & - & 1 & - & -182.160 & 3 & - & - & 1 & - & - & 2 & 3.92390 \\
\hline 4 & 2 & 1 & - & - & 1 & - & 41.0579 & 4 & 1 & - & 1 & - & - & 2 & -0.434856 \\
\hline 5 & 3 & 1 & - & - & 1 & - & -3.01225 & 5 & 2 & - & 1 & - & - & 2 & -0.177589 \\
\hline 5 & 2 & 2 & - & - & 1 & - & 0.000509436 & 4 & - & 1 & 1 & - & - & 2 & 0.657782 \\
\hline 2 & - & - & 1 & - & 1 & - & 4.95581 & 5 & 1 & 1 & 1 & - & - & 2 & -0.337476 \\
\hline 3 & 1 & - & 1 & - & 1 & - & -1.68709 & 5 & - & 2 & 1 & - & - & 2 & 0.0133100 \\
\hline 3 & - & 1 & 1 & - & 1 & - & -1.55750 & 4 & - & - & 2 & - & - & 2 & 0.308334 \\
\hline 4 & 1 & 1 & 1 & - & 1 & - & 0.0483315 & 5 & 1 & - & 2 & - & - & 2 & -0.0937625 \\
\hline 2 & - & - & - & 1 & 1 & - & 10.2464 & 5 & - & 1 & 2 & - & - & 2 & -0.0619270 \\
\hline 3 & 1 & - & - & 1 & 1 & - & -2.65305 & & & & & & & & \\
\hline
\end{tabular}

Notes. The corresponding plot is the bottom-right plot of Fig. A.1. For more details see the caption of Table A.1. 


\section{Appendix B: Mathematica code used in this work}

We present here the details of the Mathematica code used to determine and solve the system of equations to constrain the SSCmodel parameter-space. The code takes as input a grid containing the information of the simulated SSC model and the corresponding values of the observables.

For the specific case of 1RXS J101015.9 - 311909 we fixed the values of $\gamma_{\min }=100 ; \gamma_{\operatorname{Max}}=5 \times 10^{6}$ and $\alpha_{2}=4.0$, and the grid contains only the information on the remaining free parameters.

As an example, here is a part of the resulting grid used for the case of 1RXS J101015.9 - 311909 (all the values are expressed as $\log$; the grid file-name, as read by the Mathematica code is constraints_1RXSJ1010_log.dat).

\begin{tabular}{|c|c|c|c|c|c|c|c|c|c|c|c|}
\hline$\alpha_{1}$ & $\gamma_{\mathrm{br}}$ & $\mathrm{K}$ & B & $\delta$ & $\mathrm{R}$ & $v_{\text {syn }}$ & $v \mathrm{~F}_{v ; \text { syn }}$ & $v \mathrm{~F}_{v ; \mathrm{GeV}}$ & $v \mathrm{~F}_{v ; \mathrm{TeV}}$ & $\Gamma_{\mathrm{GeV}}$ & $\Gamma_{\mathrm{TeV}}$ \\
\hline & & & & & & & & -22.12 & -23.4220 & & \\
\hline & & -8.69897 & -2.30103 & 1.47577 & 15.6021 & & -17. & -21.41 & -22.5768 & & 843 \\
\hline & & -8.69897 & -2.30103 & 1.65052 & 15.6021 & 15.0000 & -16 & -20.71 & -21.74 & -2. & -3 \\
\hline 1 & 4. & -8.69897 & -2.30103 & 1.82526 & 15.6021 & 15.1111 & -16.2074 & -20.0308 & -20.9 & -1.94735 & -3.65 \\
\hline & & -8.6 & -2.3 & 000 & & & -15 & & & -1 . & -3 . \\
\hline 1 & 4.5 & -8.69897 & -2.05105 & 1.30103 & 15.6021 & 14.8 & -17.80 & -21 & -22 . & -2 & -3 \\
\hline (1) & 4. & -8.69897 & -2.05105 & 1.47577 & 15.6 & 15. & -17 & -2 & -22 . & -2 . & -3 . \\
\hline & 4. & -8.69897 & -2.05105 & 1.65052 & 15.6 & & -16 & & -21. & -1. & -3 . \\
\hline & & -8.6 & -2.05105 & 1.82526 & 15.6021 & 15.4 & -15 & & -20. & -1.87 & -3.6 \\
\hline & 4. & -8.6 & -2.05105 & 2.00000 & 15.6021 & & -15.0079 & -18.9109 & -19.5539 & -1.81029 & -3.61265 \\
\hline 1. & & -8.69897 & -1.80104 & 1.30103 & 15.6021 & 15.1111 & -17.3037 & -21.1522 & -22.3739 & -2.01313 & -3.89216 \\
\hline
\end{tabular}

\section{- Reading the grid containing model parameters and observables}

data = Import[“./constraints_1RXSJ1010_log.dat”];

$\mathrm{N} 1=$ Transpose[data][[1]]; $\gamma \mathrm{br}=$ Transpose[data][[2]];Ka = Transpose[data][[3]];B = Transpose[data][[4]];

$\delta=$ Transpose[data][[5]];size = Transpose[data][[6]]; $v$ synp = Transpose[data][[7]]; $v$ f $v$ synp = Transpose[data][[8]];

$v f v \mathrm{GeV}=$ Transpose[data][[9]];vf $v \mathrm{TeV}=$ Transpose[data][[10]];GeVSlope = Transpose[data][[11]];TeVSlope = Transpose[data][[12]];

\section{- Parametrizing the observables as a function of the model parameters}

For each observable, the best-fit formula has been estimated using the TMultiDimFit class in root (see http://root.cern. ch/ root/html/TMultiDimFit.html for details).

\section{(1) $v_{s y n}$}

vsynpregr $=$ Transpose $[\{\gamma \mathrm{br}, \boldsymbol{B}, \mathrm{Ka}, \delta$, size, $\mathrm{N} 1, v$ synp $\}]$;

fit1 = Fit[vsynpregr, $\{1, x, y, \xi, \alpha 1\},\{x, y, z, \xi, \sigma, \alpha 1\}]$

plot $1=\% / .\{x \rightarrow \gamma \mathrm{br}, y \rightarrow B, z \rightarrow \mathrm{Ka}, \xi \rightarrow \delta, \sigma \rightarrow \operatorname{size}, \alpha 1 \rightarrow \mathrm{N} 1\}$;

Show [ListPlot[Transpose[\{plot1, vsynp\}]], Plot $[x,\{x, 13.5,19\}]$, PlotRange $\rightarrow\{\{13.5,18.5\},\{13.5,18.5\}\}$, AxesOrigin $\rightarrow\{13.5,13.5\}$, AxesLabel $\rightarrow\left\{\right.$ None, " $v_{\text {syn }}$ (sampled)" $\}$, LabelStyle $\rightarrow\left\{\right.$ Bold, 11\}, Epilog $\rightarrow\left\{\right.$ Text $\left[\right.$ Style $\left[" v_{\text {syn }}(\right.$ reconstructed)", Bold, 11], $\left.\{16,12.5\}]\right\}$ PlotRangeClipping $\rightarrow$ False, ImagePadding $\rightarrow\{\{35,35\},\{40,40\}\}]$

Equarsynp $\left[\mathrm{x}_{-}, \mathrm{y}_{-}, \mathrm{z}_{-}, \xi_{-}, \sigma_{-}, \alpha 1_{-}\right]=$fit1;

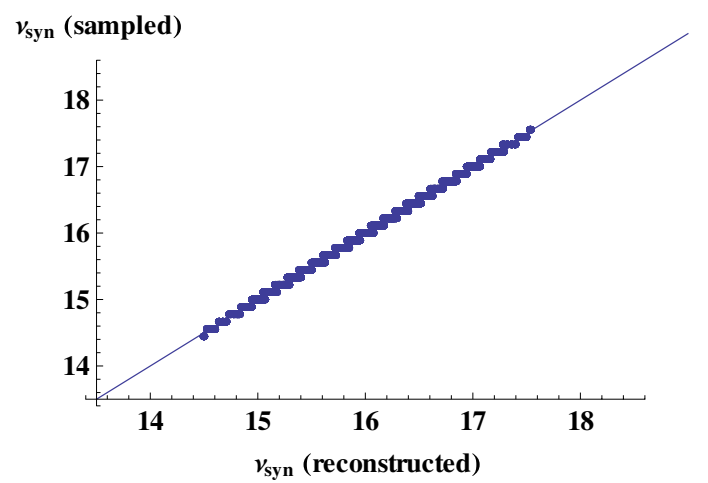


(2) $v F_{v ; \text { syn }}$

$v f v$ synpregr $=$ Transpose $[\{\gamma \mathrm{br}, B, \mathrm{Ka}, \delta$, size, N1, $v$ f $v$ synp $\}]$

fit2 = Fit [vf $v$ synpregr, $\{1, x, y, z, \xi, \sigma, \alpha 1\},\{x, y, z, \xi, \sigma, \alpha 1\}]$

plot2 $=\% / .\{x \rightarrow \gamma \mathrm{br}, y \rightarrow B, z \rightarrow \mathrm{Ka}, \xi \rightarrow \delta, \sigma \rightarrow \operatorname{size}, \alpha 1 \rightarrow \mathrm{N} 1\}$

Show [ListPlot[Transpose[\{plot2, vf vsynp\}]], Plot $[x,\{x,-20,-2\}]$, PlotRange $\rightarrow\{\{-20,-3\},\{-20,-3\}\}$, AxesOrigin $\rightarrow\{-20,-20\}$, AxesLabel $\rightarrow$ \{None, " $v \mathrm{~F}_{v \text {;syn }}$ (sampled)" $\}$, LabelStyle $\rightarrow\left\{\right.$ Bold, 11\}, Epilog $\rightarrow\left\{\right.$ Text $\left[\right.$ Style $\left[" v \mathrm{~F}_{v \text {;syn }}\right.$ (reconstructed)", Bold, 11], $\{-11,-23\}]\}$, PlotRangeClipping $\rightarrow$ False, ImagePadding $\rightarrow\{\{45,45\},\{40,40\}\}]$

Equavf $v$ synp $\left[\mathrm{x}_{-}, \mathrm{y}_{-}, \mathrm{z}_{-}, \xi_{-}, \sigma_{-}, \alpha 1_{-}\right]=$fit 2 ;

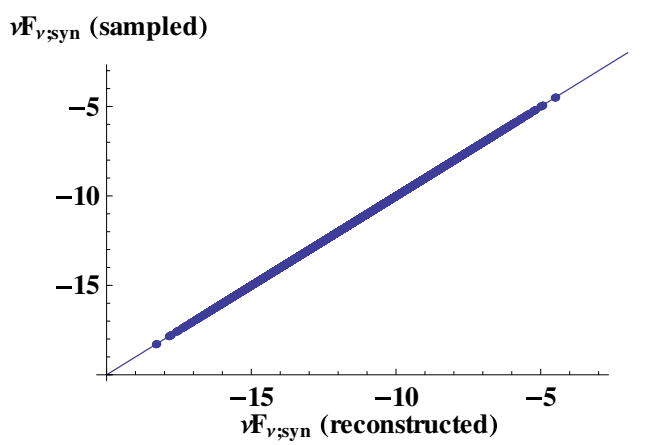

(3) $v F_{v ; G e V}$

$v \mathbf{f} v \mathrm{GeVregr}=$ Transpose $[\{\gamma \mathrm{br}, \boldsymbol{B}, \mathrm{Ka}, \delta$, size, $\mathrm{N} 1, \boldsymbol{v f} v \mathrm{GeV}\}]$

fit3 = Fit[vfvGeVregr, $\{1, x, y, z, \xi, \sigma, \alpha 1, x * x, x * y, x * \alpha 1, y * \alpha 1, x * x * \alpha 1, \xi * \alpha 1, x * y * \alpha 1, x * \xi\},\{x, y, z, \xi, \sigma, \alpha 1\}]$

plot $3=\% / .\{x \rightarrow \gamma \mathrm{br}, y \rightarrow B, z \rightarrow \mathrm{Ka}, \xi \rightarrow \delta, \sigma \rightarrow$ size, $\alpha 1 \rightarrow \mathrm{N} 1\}$

Show [ListPlot[Transpose[\{plot3, vf $v$ GeV $\}]$, Plot $[x,\{x,-28,0\}]$, PlotRange $\rightarrow\{\{-27 .,-2\},.\{-27 .,-2\}\}$, AxesOrigin $\rightarrow\{-27,-27\}$,

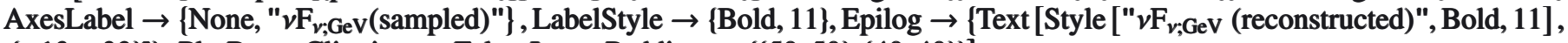
$\{-13,-32\}]\}$, PlotRangeClipping $\rightarrow$ False, ImagePadding $\rightarrow\{\{50,50\},\{40,40\}\}]$

Equavf $v \mathrm{GeV}\left[\mathrm{x}_{-}, \mathrm{y}_{-}, \mathrm{z}_{-}, \xi_{-}, \sigma_{-}, \alpha 1_{-}\right]=$fit3;

$-115.648+17.7128 x-1.5866 x^{2}+5.19307 y-0.813593 x y+1.99993 z+16.999 \alpha 1-14.1166 x \alpha 1+2.34452 x^{2} \alpha 1-4.22521 y \alpha 1+$

$1.19513 x y \alpha 1+5.74872 \xi-0.506995 x \xi+1.59946 \alpha 1 \xi+3.99993 \sigma$

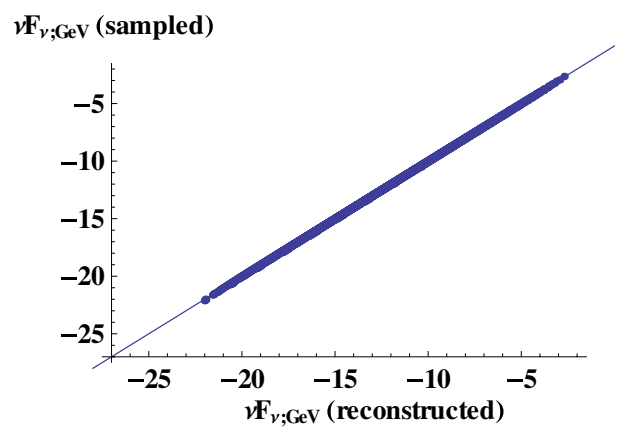

(4) $v F_{v ; T e V}$

$v \mathrm{f} v \mathrm{TeVregr}=\mathrm{Transpose}[\{\boldsymbol{\gamma} \mathrm{br}, \boldsymbol{B}, \mathrm{Ka}, \delta$, size, $\mathrm{N} 1, \boldsymbol{v f} v \mathrm{TeV}\}]$

fit4 = Fit[vfvTeVregr, $\{1, x, y, z, \xi, \sigma, \alpha 1, x * x, x * y, x * \alpha 1, x * x * y, x * \xi, x * x * y, \alpha 1 * \alpha 1 * \alpha 1, x * \alpha 1, x * x * \xi\},\{x, y, z, \xi, \sigma, \alpha 1\}]$ plot4 $=\% / .\{x \rightarrow \gamma \mathrm{br}, y \rightarrow B, z \rightarrow \mathrm{Ka}, \xi \rightarrow \delta, \sigma \rightarrow$ size, $\alpha 1 \rightarrow \mathrm{N} 1\}$

Show [ListPlot[Transpose[\{plot4, vf $v$ TeV\}]], Plot $[x,\{x,-29,-0\}]$, PlotRange $\rightarrow\{\{-28 .,-2\},.\{-28 .,-2\}\}$, AxesOrigin $\rightarrow\{-28,-28\}$, AxesLabel $\rightarrow$ \{None, " $v \mathrm{~F}_{v ; \text { TeV }}$ (sampled)" $\}$, LabelStyle $\rightarrow$ \{Bold, 11\}, Epilog $\rightarrow\left\{\right.$ Text [Style [" $v \mathrm{~F}_{v ; \text { TeV }}$ (reconstructed)", Bold, 11], $\{-15,-33\}]\}$, PlotRangeClipping $\rightarrow$ False, ImagePadding $\rightarrow\{\{50,50\},\{40,40\}\}]$

Equavf $v \mathrm{TeV}\left[\mathrm{x}_{-}, \mathrm{y}_{-}, \mathrm{z}_{-}, \xi_{-}, \sigma_{-}, \alpha 1_{-}\right]=$fit 4 ; 
$-114.123+13.7218 x-0.850289 x^{2}-4.40501 y+3.44968 x y-0.444764 x^{2} y+1.99314 z-10.8581 \alpha 1+2.34036 x \alpha 1+2.69285 \alpha 1^{3}-$ $13.1497 \xi+8.23156 x \xi-0.942506 x^{2} \xi+3.98568 \sigma$

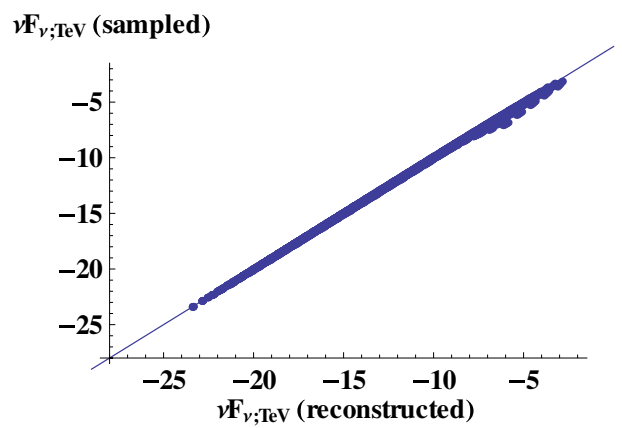

(5) $\Gamma_{\mathrm{GeV}}$

GeVSloperegr $=$ Transpose[ $[\gamma \mathrm{br}, B, \mathrm{Ka}, \delta$, size, N1, GeVSlope $\}]$;

fit5 = Fit[GeVSloperegr, $\{1, x, \alpha 1, y, \xi, x * \alpha 1, x * x, x * x * y, x * y, x * x * x, x * \xi, x * x * \xi, y * \alpha 1, x * x * x * y, x * y * \xi, \alpha 1 * \alpha 1$, $x * y * y, x * x * x * \xi, \xi * \alpha 1, x * x * y * \xi, x * x * x * x, x * x * y * y, \xi * \xi, x * x * \alpha 1\},\{x, y, z, \xi, \sigma, \alpha 1\}]$ plot5 $=\% / .\{x \rightarrow \gamma \mathrm{br}, y \rightarrow B, z \rightarrow \mathrm{Ka}, \xi \rightarrow \delta, \sigma \rightarrow \operatorname{size}, \alpha 1 \rightarrow \mathrm{N} 1\}$

Show[ListPlot[Transpose[\{plot5, GeVSlope\}]], Plot $[x,\{x,-2.6,-1.2\}]$, PlotRange $\rightarrow\{\{-2.6,-1.2\},\{-2.6,-1.2\}\}$,

AxesOrigin $\rightarrow\{-2.6,-2.6\}$, AxesLabel $\rightarrow\left\{\right.$ None, " $\Gamma_{\text {GeV }}$ (sampled)" $\}$, LabelStyle $\rightarrow\{$ Bold, 11$\}$,

Epilog $\rightarrow$ \{Text [Style [" $\Gamma_{\mathrm{GeV}}$ (reconstructed)", Bold, 11], $\left.\left.\{-1.8,-2.9\}\right]\right\}$, PlotRangeClipping $\rightarrow$ False,

ImagePadding $\rightarrow\{\{50,50\},\{40,40\}\}]$

EquaGeVSlope[x $\left.\mathbf{x}_{-}, \mathrm{y}_{-}, \mathrm{z}_{-}, \xi_{-}, \sigma_{-}, \alpha 1_{-}\right]=$fit5;

$215.795-202.197 x+67.9303 x^{2}-9.87975 x^{3}+0.528434 x^{4}-53.6426 y+35.301 x y-7.62267 x^{2} y+0.542311 x^{3} y-0.0421037 x y^{2}+$ $0.00702904 x^{2} y^{2}+41.799 \alpha 1-16.2468 x \alpha 1+1.54404 x^{2} \alpha 1-0.383771 y \alpha 1-2.36309 \alpha 1^{2}-59.7992 \xi+39.7326 x \xi-8.64464 x^{2} \xi+$ $0.621649 x^{3} \xi-0.0933089 x y \xi+0.017273 x^{2} y \xi-0.293701 \alpha 1 \xi-0.0945532 \xi^{2}$

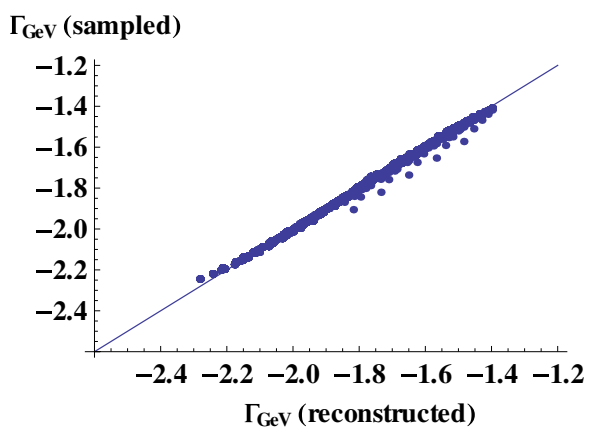

(6) $\Gamma_{\mathrm{TeV}}$

TeVSloperegr $=$ Transpose $[\{\gamma \mathrm{br}, B, \mathrm{Ka}, \delta$, size, N1, TeVSlope $\}]$;

fit6 = Fit[TeVSloperegr, $\{1, \xi, x * \xi, x * x, x, y, y * \xi, x * x * \xi, x * x * x, x * \alpha 1, x * y, x * x * y, x * y * \xi, x * z * \sigma, x * x * \alpha 1, x * y *$ $z * \sigma, x * y * z, y * z * \sigma, x * z, \xi * \alpha 1, z * \sigma, x * y * \sigma, x * \sigma, y * z, z, x * \xi * \alpha 1, y * \sigma, \sigma, \alpha 1, x * x * x * y * \xi, x * x * \xi * \xi, y * y, x * x *$ $x * \xi, x * z * z * \sigma, x * z * z, x * y * z * z, x * y * \alpha 1, x * y * z * z * \sigma, z * z * \sigma, y * z * z * \sigma, x * x * z * \sigma, x * z * \sigma * \sigma, z * z, y * z * z$ $x * x * z, x * x * \sigma, z * \sigma * \sigma, x * x * y * z, x * \sigma * \sigma, y * z * \sigma * \sigma, x * y * \sigma * \sigma, x * y * z * \sigma * \sigma, x * x * y * \sigma, x * x * y * z * \sigma, x * x *$ $y * \xi, y * \sigma * \sigma, \sigma * \sigma, x * y * y * z, x * x * x * x * \xi, y * y * z, y * y * z * \sigma, y * y * \sigma, x * x * x * \xi * \xi, x * y * y * \sigma, x * y * y * z * \sigma$, $\xi * \xi, x * x * x * y, x * x * x * x * y, x * x * x * \alpha 1, x * x * y * y, x * x * y * \alpha 1, z * \xi * \sigma, x * x * z * z, y * \xi * \alpha 1, z * \xi, x * z * z * \sigma * \sigma$, $z * z * \sigma * \sigma, x * x * z * z * \sigma, \xi * \sigma, x * x * z * \sigma * \sigma, x * x * y * \xi * \xi, x * x * \sigma * \sigma, x * z * \xi, x * x * y * z * z, x * \xi * \sigma, x * z * \xi * \sigma$, $y * z * \xi, y * z * \xi * \sigma, x * x * y * y * \xi, y * z * z * \sigma * \sigma, y * \xi * \sigma, y * \alpha 1, x * y * y * z * z, x * \xi * \xi * \xi, y * y * z * z, x * x * \xi, x * z * z *$ $z, x * x * y * \sigma * \sigma, z * z * z * \sigma, x * y * \xi * \xi, y * y * z * \sigma * \sigma, x * y * y, y * y * z * z * \sigma, z * z * z, x * x * \sigma * \sigma, x * x * x * y * y, \xi * \xi *$ $\xi, x * z * z * z * \sigma, x * y * z * \xi\},\{x, y, z, \xi, \sigma, \alpha 1\}]$

plot6 $=\% / .\{x \rightarrow \gamma \mathrm{br}, y \rightarrow B, z \rightarrow \mathrm{Ka}, \xi \rightarrow \delta, \sigma \rightarrow$ size, $\alpha 1 \rightarrow \mathrm{N} 1\}$; 
M. Cerruti et al.: Constraining the parameter space of the one-zone SSC model for GeV-TeV detected BL Lacertae objects

Show[ListPlot[Transpose[\{plot6, TeVSlope\}], PlotRange $\rightarrow$ All], Plot $[x,\{x,-5,-2.5\}]$, PlotRange $\rightarrow\{\{-5,-2.5\},\{-5,-2.5\}\}$, AxesOrigin $\rightarrow\{-5,-5\}$, AxesLabel $\rightarrow$ \{None, $" \Gamma_{\mathrm{TeV}}$ (sampled)" $\}$, LabelStyle $\rightarrow\{$ Bold, 11$\}$

$\left\{\right.$ Text [Style [" $\Gamma_{\mathrm{TeV}}$ (reconstructed)", Bold, 11], \{-3.8, -5.4\}]\}, Epilog $\rightarrow$ PlotRangeClipping $\rightarrow$ False, ImagePadding $\rightarrow\{\{50,50\},\{40,40\}\}]$

EquaTeVSlope $\left[x_{-}, y_{-}, z_{-}, \xi_{-}, \sigma_{-}, \alpha 1_{-}\right]=$fit6;

$433.763+1660.83 x-536.572 x^{2}+17.0195 x^{3}+872.651 y-83.0061 x y-83.7787 x^{2} y+5.13618 x^{3} y+0.120479 x^{4} y-209.046 y^{2}+$ $112.983 x y^{2}-18.0429 x^{2} y^{2}+1.22248 x^{3} y^{2}+937.346 z-215.023 x z-17.466 x^{2} z+50.7081 y z-64.8202 x y z+2.94899 x^{2} y z+4.10333 y^{2} z+$ $2.54005 x y^{2} z+43.3093 z^{2}-21.891 x z^{2}+1.38984 x^{2} z^{2}-25.7945 y z^{2}+2.88766 x y z^{2}-0.0347576 x^{2} y z^{2}+0.954052 y^{2} z^{2}-0.0382109 x y^{2} z^{2}-$ $2.10358 z^{3}+0.471742 x z^{3}-168.566 \alpha 1+89.7793 x \alpha 1-14.9943 x^{2} \alpha 1+0.783183 x^{3} \alpha 1+4.06062 y \alpha 1-0.0229217 x y \alpha 1-0.132376 x^{2} y \alpha 1+$ $449.564 \xi-44.9238 x \xi-76.3805 x^{2} \xi+21.1307 x^{3} \xi-1.57986 x^{4} \xi+251.194 y \xi-182.16 x y \xi+41.0579 x^{2} y \xi-3.01225 x^{3} y \xi+$ $0.000509436 x^{2} y^{2} \xi+4.95881 z \xi-1.68709 x z \xi-1.5575 y z \xi+0.0483315 x y z \xi+10.2464 \alpha 1 \xi-2.65305 x \alpha 1 \xi-0.688389 y \alpha 1 \xi-53.2164 \xi^{2}+$ $5.94909 x^{2} \xi^{2}-0.738527 x^{3} \xi^{2}+0.943224 x y \xi^{2}-0.195947 x^{2} y \xi^{2}+7.96991 \xi^{3}-1.73813 x \xi^{3}-254.494 \sigma-102.434 x \sigma+44.4274 x^{2} \sigma-$ $213.596 y \sigma+63.8761 x y \sigma+1.03778 x^{2} y \sigma+4.71668 y^{2} \sigma-1.68036 x y^{2} \sigma-122.306 z \sigma+21.0306 x z \sigma+3.83924 x^{2} z \sigma-13.7376 y z \sigma+$ $9.62879 x y z \sigma-0.221533 x^{2} y z \sigma-0.29691 y^{2} z \sigma-0.197426 x y^{2} z \sigma-7.67172 z^{2} \sigma+2.90132 x z^{2} \sigma-0.0925614 x^{2} z^{2} \sigma+2.60294 y z^{2} \sigma-$ $0.170639 x y z^{2} \sigma-0.0489805 y^{2} z^{2} \sigma+0.133559 z^{3} \sigma-0.0299422 x z^{3} \sigma-2.83619 \xi \sigma+0.931345 x \xi \sigma+0.692465 y \xi \sigma-0.341208 z \xi \sigma+$ $0.11247 x z \xi \sigma+0.0839418 y z \xi \sigma+6.46489 \sigma^{2}+4.72897 x \sigma^{2}-1.66802 x^{2} \sigma^{2}+7.24229 y \sigma^{2}-1.92839 x y \sigma^{2}-0.0907203 x^{2} y \sigma^{2}+$ $3.9239 z \sigma^{2}-0.434856 x z \sigma^{2}-0.177589 x^{2} z \sigma^{2}+0.657782 y z \sigma^{2}-0.337476 x y z \sigma^{2}+0.01331 y^{2} z \sigma^{2}+0.308334 z^{2} \sigma^{2}-0.0937625 x z^{2} \sigma^{2}-$ $0.061927 y z^{2} \sigma^{2}$

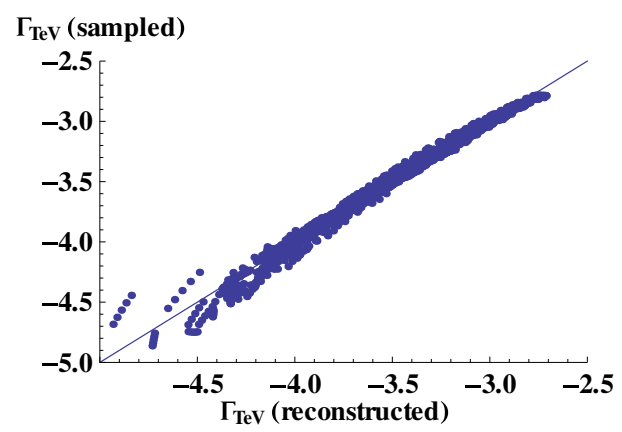

\section{- Solving the system of equations}

(1) Solutions for $\alpha 1=2.0$

Equarsynp $[x, y, z, \xi, \sigma, \alpha 1] / . \alpha 1 \rightarrow \log 10[2.0]$

Equavfvsynp $[x, y, z, \xi, \sigma, \alpha 1] / . \alpha 1 \rightarrow \log 10[2.0]$

EquavfvGeV $[x, y, z, \xi, \sigma, \alpha 1] / . \alpha 1 \rightarrow \log 10[2.0]$

Equavf $v \operatorname{TeV}[x, y, z, \xi, \sigma, \alpha 1] / \alpha 1 \rightarrow \log 10[2.0]$

EquaGeVSlope $[x, y, z, \xi, \sigma, \alpha 1] / . \alpha 1 \rightarrow \log 10[2.0]$

EquaTeVSlope $[x, y, z, \xi, \sigma, \alpha 1] / . \alpha 1 \rightarrow \log 10[2.0]$

$6.56109+1.99229 x+0.99946 y+1.00159 \xi$

$-70.4682+2.99479 x+1.99998 y+1 . z+3.99998 \xi+3.00004 \sigma$

$-110.531+13.4633 x-0.88083 x^{2}+3.92115 y-0.453824 x y+1.99993 z+6.2302 \xi-0.506995 x \xi+3.99993 \sigma$

$-117.318+14.4263 x-0.850289 x^{2}-4.40501 y+3.44968 x y-0.444764 x^{2} y+1.99314 z-13.1497 \xi+8.23156 x \xi-0.942506 x^{2} \xi+3.98568 \sigma$ $228.164-207.088 x+68.3951 x^{2}-9.87975 x^{3}+0.528434 x^{4}-53.7581 y+35.301 x y-7.62267 x^{2} y+0.542311 x^{3} y-0.0421037 x y^{2}+$ $0.00702904 x^{2} y^{2}-59.8876 \xi+39.7326 x \xi-8.64464 x^{2} \xi+0.621649 x^{3} \xi-0.0933089 x y \xi+0.017273 x^{2} y \xi-0.0945532 \xi^{2}$ 
$383.02+1687.86 x-541.085 x^{2}+17.2552 x^{3}+873.873 y-83.013 x y-83.8186 x^{2} y+5.13618 x^{3} y+0.120479 x^{4} y-209.046 y^{2}+$ $112.983 x y^{2}-18.0429 x^{2} y^{2}+1.22248 x^{3} y^{2}+937.346 z-215.023 x z-17.466 x^{2} z+50.7081 y z-64.8202 x y z+2.94899 x^{2} y z+$ $4.10333 y^{2} z+2.54005 x y^{2} z+43.3093 z^{2}-21.891 x z^{2}+1.38984 x^{2} z^{2}-25.7945 y z^{2}+2.88766 x y z^{2}-0.0347576 x^{2} y z^{2}+0.954052 y^{2} z^{2}-$ $0.0382109 x y^{2} z^{2}-2.10358 z^{3}+0.471742 x z^{3}+452.649 \xi-45.7225 x \xi-76.3805 x^{2} \xi+21.1307 x^{3} \xi-1.57986 x^{4} \xi+250.987 y \xi-182.16 x y \xi+$ $41.0579 x^{2} y \xi-3.01225 x^{3} y \xi+0.000509436 x^{2} y^{2} \xi+4.95881 z \xi-1.68709 x z \xi-1.5575 y z \xi+0.0483315 x y z \xi-53.2164 \xi^{2}+5.94909 x^{2} \xi^{2}-$ $0.738527 x^{3} \xi^{2}+0.943224 x y \xi^{2}-0.195947 x^{2} y \xi^{2}+7.96991 \xi^{3}-1.73813 x \xi^{3}-254.494 \sigma-102.434 x \sigma+44.4274 x^{2} \sigma-213.596 y \sigma+$ $63.8761 x y \sigma+1.03778 x^{2} y \sigma+4.71668 y^{2} \sigma-1.68036 x y^{2} \sigma-122.306 z \sigma+21.0306 x z \sigma+3.83924 x^{2} z \sigma-13.7376 y z \sigma+9.62879 x y z \sigma-$ $0.221533 x^{2} y z \sigma-0.29691 y^{2} z \sigma-0.197426 x y^{2} z \sigma-7.67172 z^{2} \sigma+2.90132 x z^{2} \sigma-0.0925614 x^{2} z^{2} \sigma+2.60294 y z^{2} \sigma-0.170639 x y z^{2} \sigma-$ $0.0489805 y^{2} z^{2} \sigma+0.133559 z^{3} \sigma-0.0299422 x z^{3} \sigma-2.83619 \xi \sigma+0.931345 x \xi \sigma+0.692465 y \xi \sigma-0.341208 z \xi \sigma+0.11247 x z \xi \sigma+$ $0.0839418 y z \xi \sigma+6.46489 \sigma^{2}+4.72897 x \sigma^{2}-1.66802 x^{2} \sigma^{2}+7.24229 y \sigma^{2}-1.92839 x y \sigma^{2}-0.0907203 x^{2} y \sigma^{2}+3.9239 z \sigma^{2}-$ $0.434856 x z \sigma^{2}-0.177589 x^{2} z \sigma^{2}+0.657782 y z \sigma^{2}-0.337476 x y z \sigma^{2}+0.01331 y^{2} z \sigma^{2}+0.308334 z^{2} \sigma^{2}-0.0937625 x z^{2} \sigma^{2}-0.061927 y z^{2} \sigma^{2}$

AbsoluteTiming[

sol1 = $\{$;

sol1 $=$ ParallelTable [

FindInstance[

$\{6.56109+1.99229 x+0.99946 y+1.00159 \xi==16.20-0.05+2 * 0.05 / 11 * m \& \&$

$-70.4682+2.99479 x+1.99998 y+1 . z+3.99998 \xi+3.00004 \sigma==-10.71-0.027+2 * 0.027 / 11 * l \& \&$

$-110.531+13.4633 x-0.88083 x^{2}+3.92115 y-0.453824 x y+1.99993 z+6.2302 \xi-0.506995 x \xi+3.99993 \sigma==$

$-11.88-0.10+0.20 / 11 * k \& \&$

$-117.318+14.4263 x-0.850289 x^{2}-4.40501 y+3.44968 x y-0.444764 x^{2} y+1.99314 z-13.1497 \xi+8.23156 x \xi-0.942506 x^{2} \xi$

$+3.98568 \sigma==-12.23-0.13+0.26 / 11 * j \& \&$

$228.164-207.088 x+68.3951 x^{2}-9.87975 x^{3}+0.528434 x^{4}-53.7581 y+35.301 x y-7.62267 x^{2} y+0.542311 x^{3} y-0.0421037 x y^{2}$ $+0.00702904 x^{2} y^{2}-59.8876 \xi+39.7326 x \xi-8.64464 x^{2} \xi+0.621649 x^{3} \xi-0.0933089 x y \xi+0.017273 x^{2} y \xi-0.0945532 \xi^{2} \geq-1.94 \& \&$ $228.164-207.088 x+68.3951 x^{2}-9.87975 x^{3}+0.528434 x^{4}-53.7581 y+35.301 x y-7.62267 x^{2} y+0.542311 x^{3} y-0.0421037 x y^{2}$ $+0.00702904 x^{2} y^{2}-59.8876 \xi+39.7326 x \xi-8.64464 x^{2} \xi+0.621649 x^{3} \xi-0.0933089 x y \xi+0.017273 x^{2} y \xi-0.0945532 \xi^{2} \leq-1.48 \& \&$ $383.02+1687.86 x-541.085 x^{2}+17.2552 x^{3}+873.873 y-83.013 x y-83.8186 x^{2} y+5.13618 x^{3} y+0.120479 x^{4} y-209.046 y^{2}$ $+112.983 x y^{2}-18.0429 x^{2} y^{2}+1.22248 x^{3} y^{2}+937.346 z-215.023 x z-17.466 x^{2} z+50.7081 y z-64.8202 x y z+2.94899 x^{2} y z$ $+4.10333 y^{2} z+2.54005 x y^{2} z+43.3093 z^{2}-21.891 x z^{2}+1.38984 x^{2} z^{2}-25.7945 y z^{2}+2.88766 x y z^{2}-0.0347576 x^{2} y z^{2}+0.954052 y^{2} z^{2}$ $-0.0382109 x y^{2} z^{2}-2.10358 z^{3}+0.471742 x z^{3}+452.649 \xi-45.7225 x \xi-76.3805 x^{2} \xi+21.1307 x^{3} \xi-1.57986 x^{4} \xi+250.987 y \xi$ $-182.16 x y \xi+41.0579 x^{2} y \xi-3.01225 x^{3} y \xi+0.000509436 x^{2} y^{2} \xi+4.95881 z \xi-1.68709 x z \xi-1.5575 y z \xi+0.0483315 x y z \xi-53.2164 \xi^{2}$ $+5.94909 x^{2} \xi^{2}-0.738527 x^{3} \xi^{2}+0.943224 x y \xi^{2}-0.195947 x^{2} y \xi^{2}+7.96991 \xi^{3}-1.73813 x \xi^{3}-254.494 \sigma-102.434 x \sigma+44.4274 x^{2} \sigma$ $-213.596 y \sigma+63.8761 x y \sigma+1.03778 x^{2} y \sigma+4.71668 y^{2} \sigma-1.68036 x y^{2} \sigma-122.306 z \sigma+21.0306 x z \sigma+3.83924 x^{2} z \sigma-13.7376 y z \sigma$ $+9.62879 x y z \sigma-0.221533 x^{2} y z \sigma-0.29691 y^{2} z \sigma-0.197426 x y^{2} z \sigma-7.67172 z^{2} \sigma+2.90132 x z^{2} \sigma-0.0925614 x^{2} z^{2} \sigma+2.60294 y z^{2} \sigma$ $-0.170639 x y z^{2} \sigma-0.0489805 y^{2} z^{2} \sigma+0.133559 z^{3} \sigma-0.0299422 x z^{3} \sigma-2.83619 \xi \sigma+0.931345 x \xi \sigma+0.692465 y \xi \sigma-0.341208 z \xi \sigma$ $+0.11247 x z \xi \sigma+0.0839418 y z \xi \sigma+6.46489 \sigma^{2}+4.72897 x \sigma^{2}-1.66802 x^{2} \sigma^{2}+7.24229 y \sigma^{2}-1.92839 x y \sigma^{2}-0.0907203 x^{2} y \sigma^{2}$ $+3.9239 z \sigma^{2}-0.434856 x z \sigma^{2}-0.177589 x^{2} z \sigma^{2}+0.657782 y z \sigma^{2}-0.337476 x y z \sigma^{2}+0.01331 y^{2} z \sigma^{2}+0.308334 z^{2} \sigma^{2}-0.0937625 x z^{2} \sigma^{2}$ $-0.061927 y z^{2} \sigma^{2}==-3.08-0.47+2 * 0.47 / 11 * i \& \&(\sigma-\xi+\log 10[1+0.143])<15.414 \& \&$

$\xi<2.0 \& \& \xi>1.0 \& \& y<0\},\{x, y, z, \xi, \sigma\}$, Reals, 10, WorkingPrecision $\rightarrow 10]$

$,\{m, 0,11\},\{l, 0,11\},\{k, 0,11\},\{j, 0,11\},\{i, 0,11\}] ;]$

sol1 = Flatten[sol1, 5];

xsol1 $=\{\} ;$ ysol $1=\{\} ;$ zsol $1=\{\} ; \xi$ sol $1=\{\} ; \alpha 1$ sol $1=\{\} ; \sigma$ sol $1=\{\} ;$

For $[i=1, i \leq$ Length[sol1], $i++$,

$\mathrm{xtmp}=x /$.sol1 $[[i]]$;

$\mathrm{ytmp}=y / \operatorname{sol} 1[[i]]$

$\mathrm{ztmp}=z / \cdot \operatorname{sol} 1[[i]] ;$

$\xi \operatorname{tmp}=\xi / . \operatorname{sol} 1[[i]]$

$\alpha 1 \mathrm{tmp}=2.0$;

$\sigma \operatorname{tmp}=\sigma /$ sol1 $[[i]]$;

If $[\xi \mathrm{tmp}<2.0$, AppendTo[xsol1, xtmp];

AppendTo[ysol1, ytmp];

AppendTo[zsol1, ztmp];

AppendTo[ $\xi$ soll, $\xi$ tmp];

AppendTo[ $\alpha 1$ soll, $\alpha 1 \mathrm{tmp}]$;

AppendTo[ $\sigma$ sol1, $\sigma \mathrm{tmp}] ;]$ 


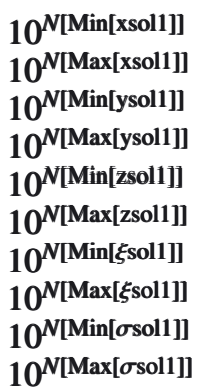

34795.8

131460 .

0.00507603

0.0408561

$9.30287 \times 10^{-10}$

$7.30166 \times 10^{-7}$

32.0741

99.5326

$4.87932 \times 10^{15}$

$1.15738 \times 10^{17}$

(2) Solutions for $\alpha 1=1.8$

\section{Equavsynp $[x, y, z, \xi, \sigma, \alpha 1] / . \alpha 1 \rightarrow \log 10[1.8]$ \\ Equarf $v \operatorname{syn}[x, y, z, \xi, \sigma, \alpha 1] / . \alpha 1 \rightarrow \log 10[1.8]$ \\ Equavf $v \operatorname{GeV}[x, y, z, \xi, \sigma, \alpha 1] / . \alpha 1 \rightarrow \log 10[1.8]$ \\ Equavf $v \operatorname{TeV}[x, y, z, \xi, \sigma, \alpha 1] / . \alpha 1 \rightarrow \log 10[1.8]$ \\ EquaGeVSlope $[x, y, z, \xi, \sigma, \alpha 1] / . \alpha 1 \rightarrow \log 10[1.8]$ \\ EquaTeVSlope $[x, y, z, \xi, \sigma, \alpha 1] / . \alpha 1 \rightarrow \log 10[1.8]$}

$6.60011+1.99229 x+0.99946 y+1.00159 \xi$

$-70.4783+2.99479 x+1.99998 y+1 . z+3.99998 \xi+3.00004 \sigma$

$-111.309+14.1093 x-0.98811 x^{2}+4.11449 y-0.50851 x y+1.99993 z+6.15702 \xi-0.506995 x \xi+3.99993 \sigma$

$-116.85+14.3192 x-0.850289 x^{2}-4.40501 y+3.44968 x y-0.444764 x^{2} y+1.99314 z-13.1497 \xi+8.23156 x \xi-0.942506 x^{2} \xi+3.98568 \sigma$ $226.311-206.345 x+68.3244 x^{2}-9.87975 x^{3}+0.528434 x^{4}-53.7405 y+35.301 x y-7.62267 x^{2} y+0.542311 x^{3} y-0.0421037 x y^{2}+$ $0.00702904 x^{2} y^{2}-59.8742 \xi+39.7326 x \xi-8.64464 x^{2} \xi+0.621649 x^{3} \xi-0.0933089 x y \xi+0.017273 x^{2} y \xi-0.0945532 \xi^{2}$ $390.733+1683.75 x-540.399 x^{2}+17.2194 x^{3}+873.687 y-83.012 x y-83.8125 x^{2} y+5.13618 x^{3} y+0.120479 x^{4} y-209.046 y^{2}+$ $112.983 x y^{2}-18.0429 x^{2} y^{2}+1.22248 x^{3} y^{2}+937.346 z-215.023 x z-17.466 x^{2} z+50.7081 y z-64.8202 x y z+2.94899 x^{2} y z+$ $4.10333 y^{2} z+2.54005 x y^{2} z+43.3093 z^{2}-21.891 x z^{2}+1.38984 x^{2} z^{2}-25.7945 y z^{2}+2.88766 x y z^{2}-0.0347576 x^{2} y z^{2}+0.954052 y^{2} z^{2}-$ $0.0382109 x y^{2} z^{2}-2.10358 z^{3}+0.471742 x z^{3}+452.18 \xi-45.6011 x \xi-76.3805 x^{2} \xi+21.1307 x^{3} \xi-1.57986 x^{4} \xi+251.018 y \xi-182.16 x y \xi+$ $41.0579 x^{2} y \xi-3.01225 x^{3} y \xi+0.000509436 x^{2} y^{2} \xi+4.95881 z \xi-1.68709 x z \xi-1.5575 y z \xi+0.0483315 x y z \xi-53.2164 \xi^{2}+5.94909 x^{2} \xi^{2}-$ $0.738527 x^{3} \xi^{2}+0.943224 x y \xi^{2}-0.195947 x^{2} y \xi^{2}+7.96991 \xi^{3}-1.73813 x \xi^{3}-254.494 \sigma-102.434 x \sigma+44.4274 x^{2} \sigma-213.596 y \sigma+$ $63.8761 x y \sigma+1.03778 x^{2} y \sigma+4.71668 y^{2} \sigma-1.68036 x y^{2} \sigma-122.306 z \sigma+21.0306 x z \sigma+3.83924 x^{2} z \sigma-13.7376 y z \sigma+9.62879 x y z \sigma-$ $0.221533 x^{2} y z \sigma-0.29691 y^{2} z \sigma-0.197426 x y^{2} z \sigma-7.67172 z^{2} \sigma+2.90132 x z^{2} \sigma-0.0925614 x^{2} z^{2} \sigma+2.60294 y z^{2} \sigma-0.170639 x y z^{2} \sigma-$ $0.0489805 y^{2} z^{2} \sigma+0.133559 z^{3} \sigma-0.0299422 x z^{3} \sigma-2.83619 \xi \sigma+0.931345 x \xi \sigma+0.692465 y \xi \sigma-0.341208 z \xi \sigma+0.11247 x z \xi \sigma+$ $0.0839418 y z \xi \sigma+6.46489 \sigma^{2}+4.72897 x \sigma^{2}-1.66802 x^{2} \sigma^{2}+7.24229 y \sigma^{2}-1.92839 x y \sigma^{2}-0.0907203 x^{2} y \sigma^{2}+3.9239 z \sigma^{2}-$ $0.434856 x z \sigma^{2}-0.177589 x^{2} z \sigma^{2}+0.657782 y z \sigma^{2}-0.337476 x y z \sigma^{2}+0.01331 y^{2} z \sigma^{2}+0.308334 z^{2} \sigma^{2}-0.0937625 x z^{2} \sigma^{2}-0.061927 y z^{2} \sigma^{2}$

\section{AbsoluteTiming[}

sol2 = $\{$; 
sol2 $=$ ParallelTable[FindInstance $[\{6.60011+1.99229 x+0.99946 y+1.00159 \xi==16.20-0.05+2 * 0.05 / 11 * m \& \&$ $-70.4783+2.99479 x+1.99998 y+1 . z+3.99998 \xi+3.00004 \sigma==-10.71-0.027+2 * 0.027 / 11 * l \& \&$

$-111.309+14.1093 x-0.98811 x^{2}+4.11449 y-0.50851 x y+1.99993 z+6.15702 \xi-0.506995 x \xi+3.99993 \sigma==$

$-11.88-0.10+0.20 / 11 * k \& \&$

$-116.85+14.3192 x-0.850289 x^{2}-4.40501 y+3.44968 x y-0.444764 x^{2} y+1.99314 z-13.1497 \xi+8.23156 x \xi-0.942506 x^{2} \xi$

$+3.98568 \sigma==-12.23-0.13+0.26 / 11 * j \& \&$

$226.311-206.345 x+68.3244 x^{2}-9.87975 x^{3}+0.528434 x^{4}-53.7405 y+35.301 x y-7.62267 x^{2} y+0.542311 x^{3} y-0.0421037 x y^{2}$

$+0.00702904 x^{2} y^{2}-59.8742 \xi+39.7326 x \xi-8.64464 x^{2} \xi+0.621649 x^{3} \xi-0.0933089 x y \xi+0.017273 x^{2} y \xi-0.0945532 \xi^{2} \geq-1.94 \& \&$ $226.311-206.345 x+68.3244 x^{2}-9.87975 x^{3}+0.528434 x^{4}-53.7405 y+35.301 x y-7.62267 x^{2} y+0.542311 x^{3} y-0.0421037 x y^{2}$ $+0.00702904 x^{2} y^{2}-59.8742 \xi+39.7326 x \xi-8.64464 x^{2} \xi+0.621649 x^{3} \xi-0.0933089 x y \xi+0.017273 x^{2} y \xi-0.0945532 \xi^{2} \leq-1.48 \& \&$ $390.733+1683.75 x-540.399 x^{2}+17.2194 x^{3}+873.687 y-83.012 x y-83.8125 x^{2} y+5.13618 x^{3} y+0.120479 x^{4} y-209.046 y^{2}$ $+112.983 x y^{2}-18.0429 x^{2} y^{2}+1.22248 x^{3} y^{2}+937.346 z-215.023 x z-17.466 x^{2} z+50.7081 y z-64.8202 x y z+2.94899 x^{2} y z$ $+4.10333 y^{2} z+2.54005 x y^{2} z+43.3093 z^{2}-21.891 x z^{2}+1.38984 x^{2} z^{2}-25.7945 y z^{2}+2.88766 x y z^{2}-0.0347576 x^{2} y z^{2}+0.954052 y^{2} z^{2}$ $-0.0382109 x y^{2} z^{2}-2.10358 z^{3}+0.471742 x z^{3}+452.18 \xi-45.6011 x \xi-76.3805 x^{2} \xi+21.1307 x^{3} \xi-1.57986 x^{4} \xi+251.018 y \xi$ $-182.16 x y \xi+41.0579 x^{2} y \xi-3.01225 x^{3} y \xi+0.000509436 x^{2} y^{2} \xi+4.95881 z \xi-1.68709 x z \xi-1.5575 y z \xi+0.0483315 x y z \xi-53.2164 \xi^{2}$ $+5.94909 x^{2} \xi^{2}-0.738527 x^{3} \xi^{2}+0.943224 x y \xi^{2}-0.195947 x^{2} y \xi^{2}+7.96991 \xi^{3}-1.73813 x \xi^{3}-254.494 \sigma-102.434 x \sigma+44.4274 x^{2} \sigma$ $-213.596 y \sigma+63.8761 x y \sigma+1.03778 x^{2} y \sigma+4.71668 y^{2} \sigma-1.68036 x y^{2} \sigma-122.306 z \sigma+21.0306 x z \sigma+3.83924 x^{2} z \sigma-13.7376 y z \sigma$ $+9.62879 x y z \sigma-0.221533 x^{2} y z \sigma-0.29691 y^{2} z \sigma-0.197426 x y^{2} z \sigma-7.67172 z^{2} \sigma+2.90132 x z^{2} \sigma-0.0925614 x^{2} z^{2} \sigma+2.60294 y z^{2} \sigma$ $-0.170639 x y z^{2} \sigma-0.0489805 y^{2} z^{2} \sigma+0.133559 z^{3} \sigma-0.0299422 x z^{3} \sigma-2.83619 \xi \sigma+0.931345 x \xi \sigma+0.692465 y \xi \sigma-0.341208 z \xi \sigma$ $+0.11247 x z \xi \sigma+0.0839418 y z \xi \sigma+6.46489 \sigma^{2}+4.72897 x \sigma^{2}-1.66802 x^{2} \sigma^{2}+7.24229 y \sigma^{2}-1.92839 x y \sigma^{2}-0.0907203 x^{2} y \sigma^{2}$ $+3.9239 z \sigma^{2}-0.434856 x z \sigma^{2}-0.177589 x^{2} z \sigma^{2}+0.657782 y z \sigma^{2}-0.337476 x y z \sigma^{2}+0.01331 y^{2} z \sigma^{2}+0.308334 z^{2} \sigma^{2}-0.0937625 x z^{2} \sigma^{2}$ $-0.061927 y z^{2} \sigma^{2}==-3.08-0.47+2 * 0.47 / 11 * i \& \&(\sigma-\xi+\log 10[1+0.143])<15.414 \& \&$

$\xi<2.0 \& \& \xi>1.0 \& \& y<0\},\{x, y, z, \xi, \sigma\}$, Reals, 10, WorkingPrecision $\rightarrow 10]$

$,\{m, 0,11\},\{l, 0,11\},\{k, 0,11\},\{j, 0,11\},\{i, 0,11\}] ;]$

sol2 = Flatten[sol2, 5];

xsol2 $=\{\} ;$ ysol $2=\{\} ;$ zsol $2=\{\} ; \xi$ sol2 $=\{\} ; \alpha 1$ sol $2=\{\} ; \sigma$ sol2 $=\{\} ;$

For $[i=1, i \leq$ Length[sol2], $i++$,

$\mathrm{xtmp}=x / . \operatorname{sol} 2[[i]]$

ytmp $=y / . \operatorname{sol} 2[[i]]$

$\mathrm{ztmp}=z /$.sol2 $[[i]]$;

$\xi \mathrm{tmp}=\xi /$. sol2[[i]];

$\alpha 1 \mathrm{tmp}=1.8$

$\sigma \operatorname{tmp}=\sigma / . \operatorname{sol} 2[[i]]$;

If $[\xi \mathrm{tmp}<2.0$, AppendTo[xsol2, xtmp];

AppendTo[ysol2, ytmp];

AppendTo[zsol2, ztmp];

AppendTo[ $\xi \mathrm{sol} 2, \xi \mathrm{tmp}]$;

AppendTo[ $\alpha 1$ sol2, $\alpha 1 \mathrm{tmp}]$;

AppendTo[ $\sigma \mathrm{sol} 2, \sigma \mathrm{tmp}] ;]$

$10^{N[\operatorname{Min}[x s o 12]]}$

$10^{N[\operatorname{Max}[\mathrm{xsol} 2]]}$

$10^{N[\operatorname{Min}[y s o 12]]}$

$10^{N[M a x[y s o l 2]]}$

$10^{N \text { Min[zsol2] }]}$

$10^{N \text { Max[zsol2]] }}$

$10^{N[M i n[\xi s o l 2]]}$

$10^{N[\mathrm{Max}[\xi \mathrm{sol} 2]]}$

$10^{N[\operatorname{Min}[\sigma \mathrm{sol} 2]]}$

$10^{N[\mathrm{Max}[\sigma \mathrm{sol} 2]]}$

34270.

112089 .

0.0076735

0.0385613

$2.30168 \times 10^{-9}$

$9.92015 \times 10^{-7}$

30.7623

99.9121

$4.5818 \times 10^{15}$

$9.66379 \times 10^{16}$

A47, page 18 of 22 
M. Cerruti et al.: Constraining the parameter space of the one-zone SSC model for GeV-TeV detected BL Lacertae objects

(3) Solutions for $\alpha 1=1.6$

Equarsynp $[x, y, z, \xi, \sigma, \alpha 1] / . \alpha 1 \rightarrow \log 10[1.6]$

Equavf $v \operatorname{synp}[x, y, z, \xi, \sigma, \alpha 1] / . \alpha 1 \rightarrow \log 10[1.6]$

Equavf $v \operatorname{GeV}[x, y, z, \xi, \sigma, \alpha 1] / . \alpha 1 \rightarrow \log 10[1.6]$

Equavf $v \operatorname{TeV}[x, y, z, \xi, \sigma, \alpha 1] / . \alpha 1 \rightarrow \log 10[1.6]$

EquaGeVSlope $[x, y, z, \xi, \sigma, \alpha 1] / . \alpha 1 \rightarrow \log 10[1.6]$

EquaTeVSlope $[x, y, z, \xi, \sigma, \alpha 1] / . \alpha 1 \rightarrow \log 10[1.6]$

$6.64374+1.99229 x+0.99946 y+1.00159 \xi$

$-70.4896+2.99479 x+1.99998 y+1 . z+3.99998 \xi+3.00004 \sigma$

$-112.179+14.8314 x-1.10804 x^{2}+4.33062 y-0.569644 x y+1.99993 z+6.0752 \xi-0.506995 x \xi+3.99993 \sigma$

$-116.316+14.1995 x-0.850289 x^{2}-4.40501 y+3.44968 x y-0.444764 x^{2} y+1.99314 z-13.1497 \xi+8.23156 x \xi-0.942506 x^{2} \xi+3.98568 \sigma$ $224.229-205.514 x+68.2454 x^{2}-9.87975 x^{3}+0.528434 x^{4}-53.7209 y+35.301 x y-7.62267 x^{2} y+0.542311 x^{3} y-0.0421037 x y^{2}+$ $0.00702904 x^{2} y^{2}-59.8592 \xi+39.7326 x \xi-8.64464 x^{2} \xi+0.621649 x^{3} \xi-0.0933089 x y \xi+0.017273 x^{2} y \xi-0.0945532 \xi^{2}$

$399.355+1679.16 x-539.632 x^{2}+17.1793 x^{3}+873.479 y-83.0108 x y-83.8058 x^{2} y+5.13618 x^{3} y+0.120479 x^{4} y-209.046 y^{2}+$ $112.983 x y^{2}-18.0429 x^{2} y^{2}+1.22248 x^{3} y^{2}+937.346 z-215.023 x z-17.466 x^{2} z+50.7081 y z-64.8202 x y z+2.94899 x^{2} y z+$ $4.10333 y^{2} z+2.54005 x y^{2} z+43.3093 z^{2}-21.891 x z^{2}+1.38984 x^{2} z^{2}-25.7945 y z^{2}+2.88766 x y z^{2}-0.0347576 x^{2} y z^{2}+0.954052 y^{2} z^{2}-$ $0.0382109 x y^{2} z^{2}-2.10358 z^{3}+0.471742 x z^{3}+451.656 \xi-45.4654 x \xi-76.3805 x^{2} \xi+21.1307 x^{3} \xi-1.57986 x^{4} \xi+251.053 y \xi-$ $182.16 x y \xi+41.0579 x^{2} y \xi-3.01225 x^{3} y \xi+0.000509436 x^{2} y^{2} \xi+4.95881 z \xi-1.68709 x z \xi-1.5575 y z \xi+0.0483315 x y z \xi-53.2164 \xi^{2}+$ $5.94909 x^{2} \xi^{2}-0.738527 x^{3} \xi^{2}+0.943224 x y \xi^{2}-0.195947 x^{2} y \xi^{2}+7.96991 \xi^{3}-1.73813 x \xi^{3}-254.494 \sigma-102.434 x \sigma+44.4274 x^{2} \sigma-$ $213.596 y \sigma+63.8761 x y \sigma+1.03778 x^{2} y \sigma+4.71668 y^{2} \sigma-1.68036 x y^{2} \sigma-122.306 z \sigma+21.0306 x z \sigma+3.83924 x^{2} z \sigma-13.7376 y z \sigma+$ $9.62879 x y z \sigma-0.221533 x^{2} y z \sigma-0.29691 y^{2} z \sigma-0.197426 x y^{2} z \sigma-7.67172 z^{2} \sigma+2.90132 x z^{2} \sigma-0.0925614 x^{2} z^{2} \sigma+2.60294 y z^{2} \sigma-$ $0.170639 x y z^{2} \sigma-0.0489805 y^{2} z^{2} \sigma+0.133559 z^{3} \sigma-0.0299422 x z^{3} \sigma-2.83619 \xi \sigma+0.931345 x \xi \sigma+0.692465 y \xi \sigma-0.341208 z \xi \sigma+$ $0.11247 x z \xi \sigma+0.0839418 y z \xi \sigma+6.46489 \sigma^{2}+4.72897 x \sigma^{2}-1.66802 x^{2} \sigma^{2}+7.24229 y \sigma^{2}-1.92839 x y \sigma^{2}-0.0907203 x^{2} y \sigma^{2}+$ $3.9239 z \sigma^{2}-0.434856 x z \sigma^{2}-0.177589 x^{2} z \sigma^{2}+0.657782 y z \sigma^{2}-0.337476 x y z \sigma^{2}+0.01331 y^{2} z \sigma^{2}+0.308334 z^{2} \sigma^{2}-0.0937625 x z^{2} \sigma^{2}-$ $0.061927 y z^{2} \sigma^{2}$

AbsoluteTiming[

sol3 = \{\}

sol3 $=$ ParallelTable[FindInstance[[6.64374 + 1.99229 $x+0.99946 y+1.00159 \xi==16.20-0.05+2 * 0.05 / 11 * m \& \&$

$-70.4896+2.99479 x+1.99998 y+1 . z+3.99998 \xi+3.000004 \sigma==-10.71-0.027+2 * 0.027 / 11 * l \& \&$

$-112.179+14.8314 x-1.10804 x^{2}+4.33062 y-0.569644 x y+1.99993 z+6.0752 \xi-0.506995 x \xi+3.99993 \sigma==$

$-11.88-0.10+0.20 / 11 * k \& \&$

$-116.316+14.1995 x-0.850289 x^{2}-4.40501 y+3.44968 x y-0.444764 x^{2} y+1.99314 z-13.1497 \xi+8.23156 x \xi-0.942506 x^{2} \xi$ $+3.98568 \sigma==-12.23-0.13+0.26 / 11 * j \& \&$

$224.229-205.514 x+68.2454 x^{2}-9.87975 x^{3}+0.528434 x^{4}-53.7209 y+35.301 x y-7.62267 x^{2} y+0.542311 x^{3} y-0.0421037 x y^{2}$ $+0.00702904 x^{2} y^{2}-59.8592 \xi+39.7326 x \xi-8.64464 x^{2} \xi+0.621649 x^{3} \xi-0.0933089 x y \xi+0.017273 x^{2} y \xi-0.0945532 \xi^{2} \geq-1.94 \& \&$ $224.229-205.514 x+68.2454 x^{2}-9.87975 x^{3}+0.528434 x^{4}-53.7209 y+35.301 x y-7.62267 x^{2} y+0.542311 x^{3} y-0.0421037 x y^{2}$ $+0.00702904 x^{2} y^{2}-59.8592 \xi+39.7326 x \xi-8.64464 x^{2} \xi+0.621649 x^{3} \xi-0.0933089 x y \xi+0.017273 x^{2} y \xi-0.0945532 \xi^{2} \leq-1.48 \& \&$ $399.355+1679.16 x-539.632 x^{2}+17.1793 x^{3}+873.479 y-83.0108 x y-83.8058 x^{2} y+5.13618 x^{3} y+0.120479 x^{4} y-209.046 y^{2}$ $+112.983 x y^{2}-18.0429 x^{2} y^{2}+1.22248 x^{3} y^{2}+937.346 z-215.023 x z-17.466 x^{2} z+50.7081 y z-64.8202 x y z+2.94899 x^{2} y z$ $+4.10333 y^{2} z+2.54005 x y^{2} z+43.3093 z^{2}-21.891 x z^{2}+1.38984 x^{2} z^{2}-25.7945 y z^{2}+2.88766 x y z^{2}-0.0347576 x^{2} y z^{2}+0.954052 y^{2} z^{2}$ $-0.0382109 x y^{2} z^{2}-2.10358 z^{3}+0.471742 x z^{3}+451.656 \xi-45.4654 x \xi-76.3805 x^{2} \xi+21.1307 x^{3} \xi-1.57986 x^{4} \xi+251.053 y \xi$ $-182.16 x y \xi+41.0579 x^{2} y \xi-3.01225 x^{3} y \xi+0.000509436 x^{2} y^{2} \xi+4.95881 z \xi-1.68709 x z \xi-1.5575 y z \xi+0.0483315 x y z \xi-53.2164 \xi^{2}$ $+5.94909 x^{2} \xi^{2}-0.738527 x^{3} \xi^{2}+0.943224 x y \xi^{2}-0.195947 x^{2} y \xi^{2}+7.96991 \xi^{3}-1.73813 x \xi^{3}-254.494 \sigma-102.434 x \sigma+44.4274 x^{2} \sigma$ $-213.596 y \sigma+63.8761 x y \sigma+1.03778 x^{2} y \sigma+4.71668 y^{2} \sigma-1.68036 x y^{2} \sigma-122.306 z \sigma+21.0306 x z \sigma+3.83924 x^{2} z \sigma-13.7376 y z \sigma$ $+9.62879 x y z \sigma-0.221533 x^{2} y z \sigma-0.29691 y^{2} z \sigma-0.197426 x y^{2} z \sigma-7.67172 z^{2} \sigma+2.90132 x z^{2} \sigma-0.0925614 x^{2} z^{2} \sigma+2.60294 y z^{2} \sigma$ $-0.170639 x y z^{2} \sigma-0.0489805 y^{2} z^{2} \sigma+0.133559 z^{3} \sigma-0.0299422 x z^{3} \sigma-2.83619 \xi \sigma+0.931345 x \xi \sigma+0.692465 y \xi \sigma-0.341208 z \xi \sigma$ $+0.11247 x z \xi \sigma+0.0839418 y z \xi \sigma+6.46489 \sigma^{2}+4.72897 x \sigma^{2}-1.66802 x^{2} \sigma^{2}+7.24229 y \sigma^{2}-1.92839 x y \sigma^{2}-0.0907203 x^{2} y \sigma^{2}+$ $3.9239 z \sigma^{2}-0.434856 x z \sigma^{2}-0.177589 x^{2} z \sigma^{2}+0.657782 y z \sigma^{2}-0.337476 x y z \sigma^{2}+0.01331 y^{2} z \sigma^{2}+0.308334 z^{2} \sigma^{2}-0.0937625 x z^{2} \sigma^{2}$ 
$-0.061927 y z^{2} \sigma^{2}==-3.08-0.47+2 * 0.47 / 11 * i \& \&(\sigma-\xi+\log 10[1+0.143])<15.414 \& \&$ $\xi<2.0 \& \& \xi>1.0 \& \& y<0\},\{x, y, z, \xi, \sigma\}$, Reals, 10$],\{m, 0,11\},\{l, 0,11\},\{k, 0,11\},\{j, 0,11\},\{i, 0,11\}] ;$ ] sol3 = Flatten[sol3, 5];

xsol $3=\{\} ;$ ysol $3=\{\} ;$ zsol $3=\{\} ; \xi$ sol $3=\{\} ; \alpha 1$ sol $3=\{\} ; \sigma$ sol $3=\{\} ;$

For $[i=1, i \leq$ Length[sol3], $i++, \mathrm{xtmp}=x /$ sol3[[i]];

ytmp $=y /$. sol3[[i] $]$;

$\mathrm{ztmp}=z / \cdot \operatorname{sol} 3[[i]]$

$\xi \operatorname{tmp}=\xi / . \operatorname{sol} 3[[i]]$

$\alpha 1 \mathrm{tmp}=1.6$

$\sigma \operatorname{tmp}=\sigma / . \operatorname{sol} 3[[i]]$

If $\xi \xi \mathrm{tmp}<2.0$, AppendTo[xsol3, xtmp];

AppendTo[ysol3, ytmp];

AppendTo[zsol3, ztmp];

AppendTo[ $\xi$ sol3, $\xi \mathrm{tmp}]$;

AppendTo[ $[\alpha 1$ sol3, $\alpha 1$ tmp];

AppendTo[ $\sigma$ sol $3, \sigma \mathrm{tmp}] ;]$

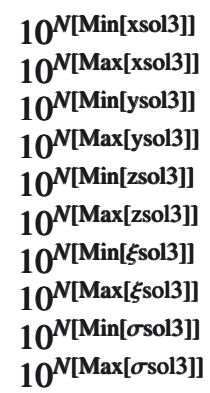

\section{- Histogram plotting}

Histogram[\{Flatten[ $N$ [xsol3]], Flatten[ $N[\mathrm{xsol} 2]]$, Flatten[ $N[\mathrm{xsol1}]]\}, 14$, PlotRange $\rightarrow$ All, ChartLayout $\rightarrow$ "Stacked"] Histogram[[Flatten[ $N[$ ysol3]], Flatten[ $N$ [ysol2]], Flatten[ $N$ [ysol1]]\}, 11, PlotRange $\rightarrow$ All, ChartLayout $\rightarrow$ "Stacked"] Histogram[\{Flatten[ $N[z s o 13]]$, Flatten[ $N$ [zsol2]], Flatten[ $N$ [zsol1]]\}, 9, PlotRange $\rightarrow$ All, ChartLayout $\rightarrow$ "Stacked"] Histogram[\{Flatten[ $N[\xi \operatorname{sol} 3]]$, Flatten[ $N[\xi$ sol2]], Flatten[ $N[\xi$ sol1]]\}, 9, PlotRange $\rightarrow$ All, ChartLayout $\rightarrow$ "Stacked"] Histogram[\{Flatten[ $N[\sigma$ sol3]], Flatten[ $N[\sigma$ sol2]], Flatten[ $N[\sigma$ sol1] $]\}, 10$, PlotRange $\rightarrow$ All, ChartLayout $\rightarrow$ "Stacked"] Histogram[[Flatten[ $N[\alpha 1$ sol3]], Flatten[ $N[\alpha 1$ sol2]], Flatten[ $N[\alpha 1$ sol1]]\}, 8, PlotRange $\rightarrow$ All, ChartLayout $\rightarrow$ "Stacked"]

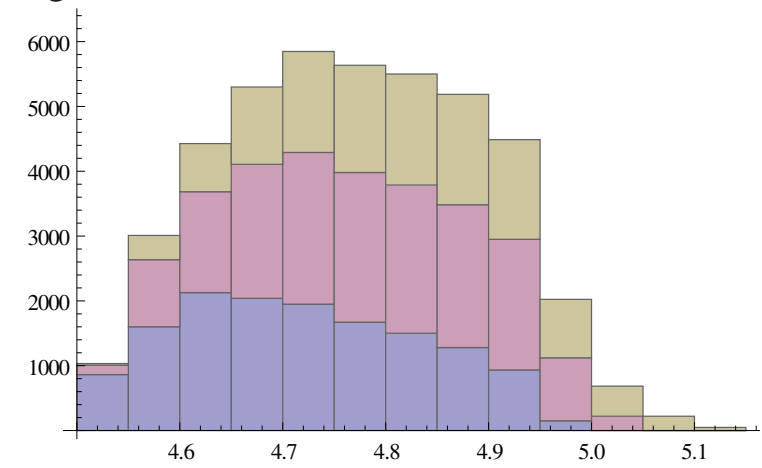


M. Cerruti et al.: Constraining the parameter space of the one-zone SSC model for GeV-TeV detected BL Lacertae objects
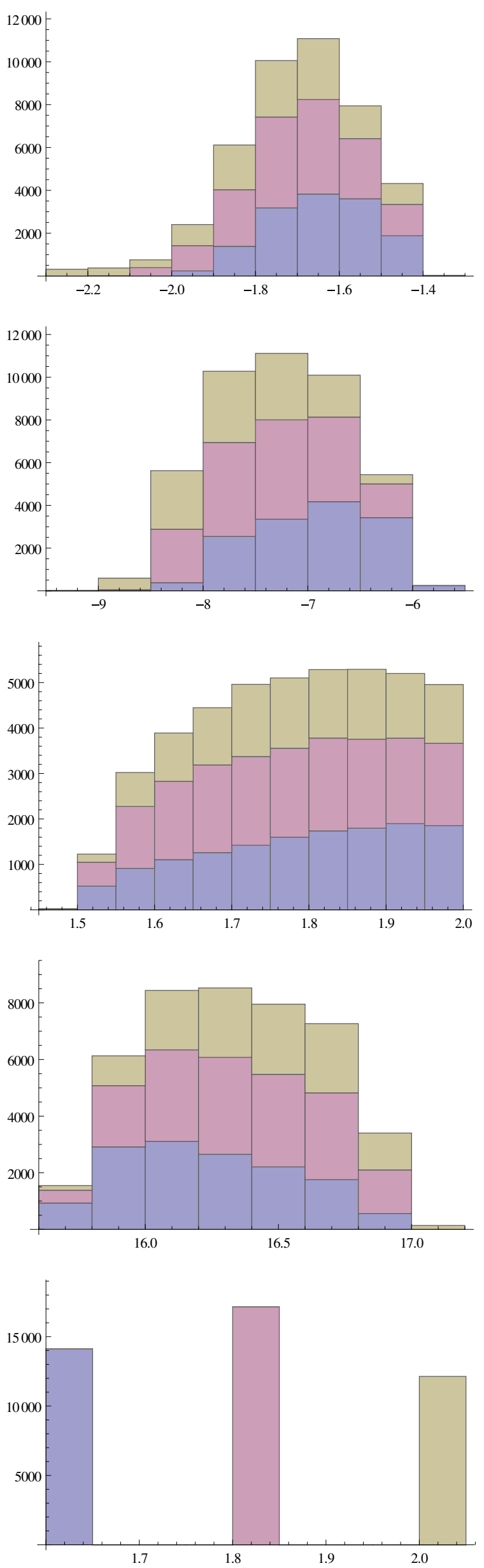


\section{- Exporting the solutions as text file}

xtot $1=N$ [xsol1];

xtot2 $=$ Flatten[AppendTo[xtot1, $N$ [xsol2]]];

xtot3 = Flatten[AppendTo[xtot2, $N[$ xsol3]]];

ytot1 $=N$ [ysol1]

ytot2 = Flatten[AppendTo[ytot1, $N$ [ysol2]]];

ytot3 = Flatten[AppendTo[ytot2, $N[$ ysol3]]];

ztot1 $=N$ [zsol1 $]$;

ztot2 = Flatten[AppendTo[ztot, $N$ [zsol2]]];

ztot3 = Flatten[AppendTo[ztot2, $N[\mathrm{zsol} 3]]]$;

$\xi$ tot $1=N[\xi$ sol1 $] ;$

$\xi$ tot2 $=$ Flatten[AppendTo[ $\xi$ tot $1, N[\xi$ sol2]]];

$\xi$ tot3 = Flatten[AppendTo[ $\xi$ tot2, $N[\xi$ sol3]]];

$\sigma$ tot $1=N[\sigma$ sol1 $] ;$

$\sigma$ tot2 $=$ Flatten[AppendTo[ $\sigma$ tot $1, N[\sigma$ sol2 $]]]$;

$\sigma$ tot3 = Flatten[AppendTo[ $\sigma$ tot $2, N[\sigma$ sol3 $]]]$;

$\alpha 1$ tot $1=N[\alpha 1$ sol1 $] ;$

$\alpha 1$ tot $2=$ Flatten[AppendTo[ $\alpha 1$ tot $1, N[\alpha 1$ sol2] $]$;

$\alpha 1$ tot 3 = Flatten[AppendTo[ $\alpha 1$ tot $2, N[\alpha 1$ sol3] $]$;

Put[xtot3, "gamma_break_solutions.dat"]

Put[ytot3, "B_solutions.dat"]

Put[ztot3, "K_solutions.dat"]

Put[รtot3, "delta_solutions.dat"]

Put[otot3, "R_solutions.dat"]

Put[ $\alpha 1$ tot3, "alpha1_solutions.dat"] 